\title{
Dust outflows from quiescent spiral disks
}

\author{
P.B. Alton ${ }^{1}$, R.J. Rand ${ }^{2}$, E.M. Xilouris ${ }^{3}$, S. Bevan ${ }^{1}$, A.M. Ferguson ${ }^{4}$, J.I. Davies ${ }^{1}$, and S. Bianchi ${ }^{1}$ \\ 1 Department of Physics \& Astronomy, University of Wales, P.O. Box 913, Cardiff CF2 3YB, UK \\ 2 University of New Mexico, Department of Physics and Astronomy, 800 Yale Boulevard, NE, Albuquerque, NM 87131, U.S.A. \\ 3 Foundation for Research \& Technology-Hellas, P.O. Box 1527, 71110 Heraklion, Crete, Greece \\ ${ }^{4}$ Institute of Astronomy, University of Cambridge, Madingley Road, Cambridge CB3 0HA, UK
}

Received December 13; accepted April 26, 2000

\begin{abstract}
We have conducted a search for "dust chimneys" in a sample of 10 highly-inclined spiral galaxies $\left(i=86-90^{\circ}\right)$ which we had previously observed in the $\mathrm{H} \alpha$ emission line (Rand 1996). We have procured $B$-band CCD images for this purpose and employed unsharp-masking techniques to accentuate the structure of the dust lane. A scattering+absorption radiation transfer model enabled us to separate 5 galaxies from the sample which are sufficiently inclined $\left(i>87^{\circ}\right)$ for us to reliably identify and quantify dust clouds residing at over 2 scale-heights above the disk. Three of these galaxies possess numerous curvi-linear chimney structures stretching up to $2 \mathrm{kpc}$ from the midplane and the fraction of total galactic dust contained in such structures is of order $1 \%$. Optical extinction offers a lower limit to the amount of dust contained in the extraplanar layer but, by examining the transparent submm thermal emission from NGC 891, we fix an upper limit of $5 \%$. Our results are consistent with a similar recent study by Howk \& Savage (1999) which indicates that about half of quiescent spiral disks possess detectable dust chimneys.
\end{abstract}

We have compared our optical images with the corresponding $\mathrm{H} \alpha$ emission-line radiation. We do not find a detailed spatial correspondance between dust chimneys and either sites of recent star-formation or the extraplanar diffuse ionized gas. This is somewhat surprising given that FIR-bright galaxies, such as M 82, are known to entrain dust at the working surface of the starburst-driven outflow (traced in $\mathrm{H} \alpha$ ). It is possible a global correlation exists, with disks experiencing overall higher rates of starformation also possessing the greatest number of chimneys. This may indicate a timescale difference between the two phenomena with the $\mathrm{H} \alpha$ phase lasting $\sim 10^{6} \mathrm{yr}$ but chimneys requiring $\sim 10^{7}$ yr to form.

Additionally, we have investigated the edge-on disk NGC 55 which, being ten times closer than galaxies in

Send offprint requests to: P.B. Alton, e-mail: paul.alton@astro.cf.ac.uk our main sample, allows us to examine in greater spatial detail the relationship between chimneys and recent starformation. Our discussion touches upon high latitude dust and supershells observed in the Milky Way. We rule out quiescent disks as prolific sources of intergalactic grains and metals but note that the rate at which dust is expelled from the main dust layer is comparable to the rate at which it is produced by disk stars (suggesting that it may be an important regulatory process).

Key words: dust, extinction — galaxies: spiral — galaxies: ISM — galaxies: individual: NGC 891 — infrared: galaxies

\section{Introduction}

The notion that certain galaxies expel dust from their main gas layer into the lower halo $(z$-height $>1 \mathrm{kpc})$ has only been recognized quite recently. For example, the starburst-driven expulsion of ionized gas from the centre of far-infrared (FIR) bright galaxies, like M 82, is now believed to entrain denser, neutral material forming a "vertical" network of gas and dust streamers at the working surface of the expansion (Hughes et al. 1994; Alton et al. 1999a; Phillips 1993; Ichikawa et al. 1994). For nonstarburst or quiescent galaxies ${ }^{1}$, the occurence of such structures, or indeed any kind of protuberance from the notional dust lane, has rarely been remarked upon in the literature and, if so, usually en passant. Sofue et al. (1994) carried out a dedicated study of the dust lane in nearby NGC 253 and catagorize an ensemble of filaments and loops surmounting the traditional dust layer (scale-size $\leq 1 \mathrm{kpc}$ ). This galaxy, however, is known to be experiencing a starburst phase similar to that of $\mathrm{M} 82$ (e.g. McCarthy et al. 1987) and, furthermore, its inclined

\footnotetext{
1 In Sect. 2 we define quiescent systems as having a FIR-to-blue luminosity ratio of order unity or less.
} 
aspect $\left(i \simeq 80^{\circ}\right)$ makes the actual height of such features above the stellar midplane uncertain ${ }^{2}$.

Keppel et al. (1991) noted an abundance of "dust chimneys" leaking out to heights of $1-2 \mathrm{kpc}$ above the main absorption layer in NGC 891 (so-called "fishbone effect"). This nearby, perfectly edge-on disk has recently come under closer scrutiny by Howk \& Savage (1997; HS97) who used optical images of sub-arcsecond resolution to isolate and describe the extraplanar material. In another approach, Alton et al. (1998a, 1999b) mapped the submillimeter (submm) thermal emission from the dark lane in NGC 891 in order to try and infer properties for the extraplanar dust structures (see also Israel et al. 1999). Whilst the submm observations remain difficult to interpret, the conclusion from the optical study was that high-latitude ( $z$-height $>0.4 \mathrm{kpc})$ dust features have a $B$-band opacity of $0.5-1$ and comprise $\sim 1 \%$ of the gas associated with the disk (assuming a ratio of gas-to-dust similar to the solar neighborhood).

Given that NGC 891 is known to have a reasonably active halo, with both neutral and ionized gas extending up to several kpc from the midplane (Dettmar 1990; Swaters et al. 1997; Rand et al 1990), it is not obvious whether dust chimneys are commonplace amongst disk galaxies. Indeed, we have shown (Rand 1996; hereafter R96) that NGC 891 possesses one of the brightest ionized gas extraplanar layers amongst nearby edge-on galaxies suggesting that the transfer of energy and mass between disk and halo is very pronounced in this case. Using the WIYN imager, Howk \& Savage (1999; hereafter HS99) recently extended their optical observations to 7 edge-on systems, of which 5 were found to contain extraplanar dust. At the same time that the HS99 study was still in progress, we procured $B$-band images of the 10 galaxies which we had already observed in the $\mathrm{H} \alpha$ emission line (R96). The objective for both groups is to establish how commonplace dust chimneys are amongst spiral galaxies and to clarify how extraplanar dust might relate to recent star-formation in the disk below. The notion that dust chimneys may be connected with young stars stems from the observation, in starburst galaxies, that massive stars and supernovae collectively lift dust out of the main disk (an effect known as the "superwind"; Chevalier \& Clegg 1985; Heckman et al. 1990).

There is significant overlap between the galaxies presented here and those investigated by HS99. Our sample is essentially fixed by those disks we had already imaged in $\mathrm{H} \alpha$ and, furthermore, there are perhaps only a dozen or so edge-on spirals which are sufficiently close to allow detailed study of extraplanar structures. Although HS99 preside over higher resolution optical images than ourselves we employ a radiative transfer model to separate more reliably the standard dust layer from putative extra-

\footnotetext{
${ }^{2}$ Throughout this paper we adopt $i=90^{\circ}$ for a perfectly edge-on galaxy.
}

planar features (Sect. 3). This is important because if the object of interest is not perfectly edge-on like NGC 891, then signficant confusion arises between structure within the disk and features which truly extend out of the disk (indeed, for this reason, we reject from the final analysis some of the galaxies which HS99 label as manifesting extraplanar dust). After using unsharp-masking techniques to locate unequivocal extraplanar features we relate the occurence of such structures to $\mathrm{H} \alpha$ emission emanating from both the corresponding halo region and the disk below (Sect. 4.3). For a number of reasons, tracing extraplanar dust by extinction alone is likely to give a lower estimate to the amount of material existing outside the main dust/gas layer. Consequently, we analyse the vertical distribution of submm emission detected in NGC 891 (at the transparent wavelengths of 450 and $850 \mu \mathrm{m}$ ) in order to constrain the maximum amount of extraplanar material that may be present (Sect. 5.1).

An important corollary of this work is the rate of mass loss from quiescent disks (Sect. 6.4) and whether this is likely to be a rich source of gas and dust for the intergalactic medium (IGM). Numerically, quiescent spirals constitute one of the primary galaxy types both in the field and in rich clusters (Dressler 1980). Potentially, therefore, any seepage from the main stellar disk may contaminate the medium between galaxies with both metals and lightabsorbing particulates (Wiebe et al. 1999). The latter, when dispersed over a large volume, are particularly effective at both blocking and reddening light passing through the IGM (Heisler \& Ostriker 1988; Masci \& Webster 1999; Alton et al. 1999b). This effect would explain the preferential optical-reddening of radio quasars viewed through the IGM of nearby clusters (Webster et al. 1995; Fall \& Pei 1993; Malhotra 1997) and could eventually be implicated in the relatively large scale-size $(\sim 80 \mathrm{kpc})$ inferred for QSO absorption-line systems (Lanzetta et al. 1987). At the very least, even if dust chimneys do not possess sufficient kinetic energy to liberate grains completely from the host disk, they are still likely to play an important role in the circulation of metal-rich, dusty gas around the ISM. This mixing dynamo would constitute a key process in the chemical evolution and star-formation history of galactic disks (Edmunds 1996).

\section{Object selection and observations}

Our galaxy sample is comprised of 9 edge-on spirals for which we already possess deep images in the $\mathrm{H} \alpha$ emissionline $(\lambda=6563 \AA)$. These galaxies were originally selected on the basis of their appearing nearby (apparent diameter $5-10^{\prime}$ ) and having inclinations within a few degrees of edge-on (R96). We added to this NGC 891 which has been extensively studied by one of us (Rand 1998; Rand et al. 1990) and, as already indicated, represents the archetypal or prototypal "chimney galaxy". It is important to 
emphasize that our main sample has not been chosen on the basis of either high emission-line brightness or enhanced far-infrared (FIR) luminosity. It is expected then to preclude any bias towards active or starburst galaxies, making our results far-reaching in the sense that they will apply to typical spiral disks. We use both the FIR luminosity and the ratio of FIR-to-blue luminosities to check the level of recent star-formation in our sample. Both quantities are known to assume high values in systems displaying starburst activity (Heckman et al. 1990; Soifer et al. 1997; Alton et al. 1998b). High Resolution IRAS maps (HiRes) at 60 and $100 \mu \mathrm{m}$ were obtained on request from the Infrared Processing and Analysis Center at Caltech (IPAC). These data, in conjunction with optical photometry from the RC3 (de Vaucouleurs et al. 1991), allowed us to infer mean values of 4.6 $10^{9} L_{\odot}$ and 1.1 for the FIR luminosity and $L_{\mathrm{FIR}}$-to- $L_{B}$ ratio respectively (Table 1 ). These quantities correspond much better to the properties of nearby, optically-selected IRAS galaxies ( $510^{9} L_{\odot}$ and 0.6 respectively) rather than to starburst disks ( $510^{10}$ and $\sim 10$ respectively), and confirms that our sample consists chiefly of quiescent rather than starburst galaxy types (Soifer et al. 1987; Heckman et al. 1990). We can be less sure that NGC 891 complies with the original selection criteria. It is indeed one of the nearest examples of a large edge-on disk but an extensive history of observation indicates that it possesses a fairly active halo which is probably fed by a disk harbouring fairly vigorous star-formation. The FIR properties of NGC 891 indicate somewhat enhanced levels of recent star-formation over "normal" disks but Table 1 shows that this galaxy is much better classified as a quiescent rather than a starburst system.

In order to carry out a rigorous search for extraplanar extinction features we required deep, optical images for our sample. For a few of the galaxies in Table 1 (NGC 891, NGC 4013, NGC 5907), we were already in possession of deep $B, V$ and $R$ images taken with the 1.3-m telescope at Skinakas Observatory, Greece (Xilouris et al. 1999). For the remainder, we carried out $B$-band imaging at the Kitt Peak 0.6-m telescope during April 1998 but bad weather prevented us from obtained images with sufficient signal. To complete coverage of the sample we accessed data from the Isaac Newton Group (ING) archive at Cambridge. Table 2 summarizes the sources and important properties of the $B$-band data. Since our sample was not observed under constant seeing conditions using an identical instrumental set-up we will examine, at a later stage, the effect that varied observing conditions might have on our sensitivity to extraplanar dust.

The archive data were reduced in the standard manner using the STARLINK software package CCDPACK to perform debiasing, flatfielding and sky subtraction etc. We also removed the effects of cosmic rays by aligning and median stacking multiple exposures of the same object. It was not necessary to photometrically calibrate any of the images. Neither the recognition of dust chimneys nor the calculation of optical depth for such features relies on the absolute brightness of the galaxy but solely on the relative difference in brightness between attenuated regions and adjacent, unextinguished regions.

\section{Image processing}

In order to classify features as "extraplanar" (i.e. as existing outside the standard absorption lane) it is necessary to define the height and inclination of the stellar and dust layers within each of our objects. To this end, we used the radiation transfer model of Xilouris et al. (1997, 1998, $1999)$ to fit the observed $B$-band surface photometry with a model galaxy consisting of an exponential stellar disk, a $R^{\frac{1}{4}}$ bulge and an exponential dust distribution. This model carries out a pixel-to-pixel comparison between the real and simulated object in order to fit scale-heights (z-direction) and scale-lengths (radial direction) to both the stellar and dust disks and to determine the optical depth through the centre of the galaxy (as seen face-on). Consistent with an environment where stars and dust are mixed, the simulation takes account of photon scattering, through the Henyey-Greenstein phase function (Henyey \& Greenstein 1941; Bohren \& Huffman 1983), as well as radiation absorption. Our confidence in the Xilouris model is significantly boosted by the fact that, in the past, the output parameters from this technique have been highly consistent across several optical and NIR wavebands. Thus in NGC 891, for example, the radial scale-length for the grain distribution has been calculated to be $8.1 \mathrm{kpc}$ in the $V$-band whilst an independent determination in other filters $(K, J, I, B)$ strays by only $5-10 \%$ from this value (Xilouris 1998). The opacity derived from the Xilouris model, which is determined independently at each wavelength, is also remarkably consistent with a Galactic extinction law for all 7 edge-on disks previously analysed (Xilouris et al. 1999). Table 3 shows the properties we derive for each of the galaxies appearing in the sample. For NGC 5023 and UGC 4278 the irregular, clumpy appearance of the disk meant that we could not parameterize the dust layer satisfactorily with an exponential model. In these cases, we will assume that the grains are distributed with a scale-height $0.51 \times$ that of the stars, and a scale-length $1.2 \times$ that of the stars (in line with the mean properties of the other galaxies in Table 3). The surface photometry of NGC 4762 is completely smooth suggesting that this SO galaxy contains no absorption layer whatsoever. Therefore, no dust parameters are listed for this object.

A difficulty that we have encountered early on in the analysis is that, for objects within the sample that are not perfectly edge-on, there may exist line-of-sight confusion between structure within the plane and features that genuinely extend "vertically" out of the plane. Consequently, 
Table 1. Basic properties of the galaxy sample. The object position, spiral type and distance are taken from Rand (1996). The blue magnitude, $B_{\text {corr }}$, is extracted from the RC3 and is corrected for Milky Way extinction only (i.e. no account of internal extinction has been taken). $L_{\mathrm{FIR}}$ is the far-infrared luminosity obtained from integrating 60 and $100 \mu \mathrm{m}$ flux densities in the HiRes IRAS data. The FIR-to-blue luminosity ratio follows the conventional definition of Rice et al. (1988)

$\begin{array}{lcccccccc}\text { Galaxy } & \text { Other Designation } & \text { Type } & \begin{array}{c}\text { R.A. } \\ (\mathrm{J} 2000)\end{array} & \begin{array}{c}\text { Dec. } \\ (\mathrm{J} 2000)\end{array} & \begin{array}{c}D \\ (\mathrm{Mpc})\end{array} & \begin{array}{c}B_{\text {corr }} \\ (\mathrm{mag})\end{array} & \begin{array}{c}L_{\mathrm{FIR}} \\ \left(10^{10} L_{\odot}\right)\end{array} & \begin{array}{c}L_{\mathrm{FIR}} \\ L_{\mathrm{B}}\end{array} \\ \text { NGC 891 } & \text { UGC 01831 } & \mathrm{Sb} & 022233.0 & +422056 & 9.5 & 10.5 & 1.4 & 3.2 \\ \text { UGC 4278 } & \text { IC 2233 } & \mathrm{Sd} & 081358.8 & +454436 & 10.6 & 12.9 & 0.014 & 0.23 \\ \text { NGC 4013 } & \text { UGC 06963 } & \mathrm{Sbc} & 115831.1 & +435650 & 17.0 & 12.2 & 0.47 & 1.6 \\ \text { NGC 4217 } & \text { UGC 07282 } & \mathrm{Sb} & 121550.7 & +470537 & 17.0 & 12.0 & 0.80 & 2.4 \\ \text { NGC 4302 } & \text { UGC 07418 } & \mathrm{Sc} & 122142.3 & +143552 & 16.8 & 12.4 & 0.22 & 0.93 \\ \text { NGC 4762 } & \text { UGC 08016 } & \mathrm{SO} & 125256.3 & +111348 & 16.8 & 11.1 & <0.025 & <0.033 \\ \text { NGC 5023 } & \text { UGC 08286 } & \mathrm{Scd} & 131212.0 & +440220 & 6 & 12.9 & 0.0049 & 0.25 \\ \text { NGC 5746 } & \text { UGC 09499 } & \mathrm{Sb} & 144456.4 & +015716 & 29.4 & 11.2 & 0.66 & 0.29 \\ \text { NGC 5907 } & \text { UGC 09801 } & \mathrm{Sc} & 151554.0 & +561945 & 14.9 & 11.1 & 0.76 & 1.25 \\ \text { UGC 10288 } & - & \mathrm{Sc} & 161425.0 & -001225 & 31.5 & 13.8 & 0.26 & 1.13\end{array}$

Table 2. B-band observations for the galaxy sample

$\begin{array}{lllcc}\text { Galaxy } & \text { Telescope } & \text { Observing date } & \begin{array}{c}\text { Exposure } \\ (\min )\end{array} & \begin{array}{c}\text { Seeing FWHM } \\ \text { (arcsec) }\end{array} \\ & & & & \\ \text { NGC 891 } & \text { Skinakas 1.3 m } & \text { October 1996 } & 80 & 1.4 \\ \text { UGC 4278 } & \text { INT 2.5 m } & \text { December 1995 } & 10 & 2.5 \\ \text { NGC 4013 } & \text { Skinakas 1.3 m } & \text { June 1995 } & 140 & 1.2 \\ \text { NGC 4217 } & \text { JKT 1.0 m } & \text { April 1999 } & 90 & 1.2 \\ \text { NGC 4302 } & \text { INT 2.5 m } & \text { April 1991 } & 17 & 1.3 \\ \text { NGC 4762 } & \text { JKT 1.0 m } & \text { March 1994 } & 90 & 1.6 \\ \text { NGC 5023 } & \text { JKT 1.0 m } & \text { April 1993 } & 60 & 2.0 \\ \text { NGC 5746 } & \text { INT 2.5 m } & \text { June 1990 } & 8.3 & 1.8 \\ \text { NGC 5907 } & \text { Skinakas 1.3 m } & \text { May 1997 } & 30 & 1.8 \\ \text { UGC 10288 } & \text { JKT 1.0 m } & \text { April 1999 } & 90 & 1.2\end{array}$

we dismissed from the final sample objects with inclinations $i \leq 87^{\circ}$. This rather stringent condition arose from the fact that a disk inclined at $87^{\circ}$ to the lineof-sight will project vertically $h_{\mathrm{D}} \times \operatorname{Cos}\left(87^{\circ}\right)$ onto the plane of the sky ( $h_{\mathrm{D}}$ being the dust scale-length). This is then comparable to the grain scale-height, $z_{\mathrm{D}}$, indicating that confusion is likely to arise. The $i \leq 87^{\circ}$ criterion reduces our sample to only half its original size (now NGC 891, NGC 4013, NGC 4302, NGC 4762 and UGC 4278 remain). The reduction in sample size is unfortunate but it is important to be certain that the "vertical" extinction features that we subsequently identify truly reside outside the notional dust lane.

In the past, authors have been less stringent in discriminating between features termed "extraplanar" and the main absorption lane. The description of "vertical filaments and loops" in NGC 253, for example, is based on a disk that is inclined by $30^{\circ}$ to the line-of-sight (Sofue et al. 1994). HS97 do not appear to state a criterion for separation of extraplanar dust from the central dark lane in NGC 891. For their larger sample (HS99) these authors use a generic height of 400 pc (projected) as the boundary between high-lattitude dust and the main extinction layer. This is close to twice the average scale-height determined for our own sample based on radiation transfer (Table 3). However, the projected height of the main dust layer will vary from galaxy to galaxy depending on inclination and, in contrast to previous authors, we feel obliged to take account of line-of-sight effects (see below). Notably, 2 galaxies which HS99 label as manifesting extraplanar dust are rejected from our final edge-on sample because they are believed to be too inclined for reliable separation of projected in-plane structure from bonafide vertical structures (NGC 5907 and NGC 4217).

We use a projected $z$-height of $2 \times l$ to discriminate extraplanar extinction from the main absorption layer, where:

$l=h_{\mathrm{D}} \operatorname{Cos}(i)+z_{\mathrm{D}}$.

Here, $i$ is the inclination of the disk, whilst $h_{\mathrm{D}}$ and $z_{\mathrm{D}}$ are the dust scale-length and scale-height, respectively, as listed in Table 3 . A height of $2 \times l$ ensures that only structures extending to at least twice the scale-height of the main dust layer are recognized as extraplanar. The first term in Eq. (1) takes account of the fact that projection 

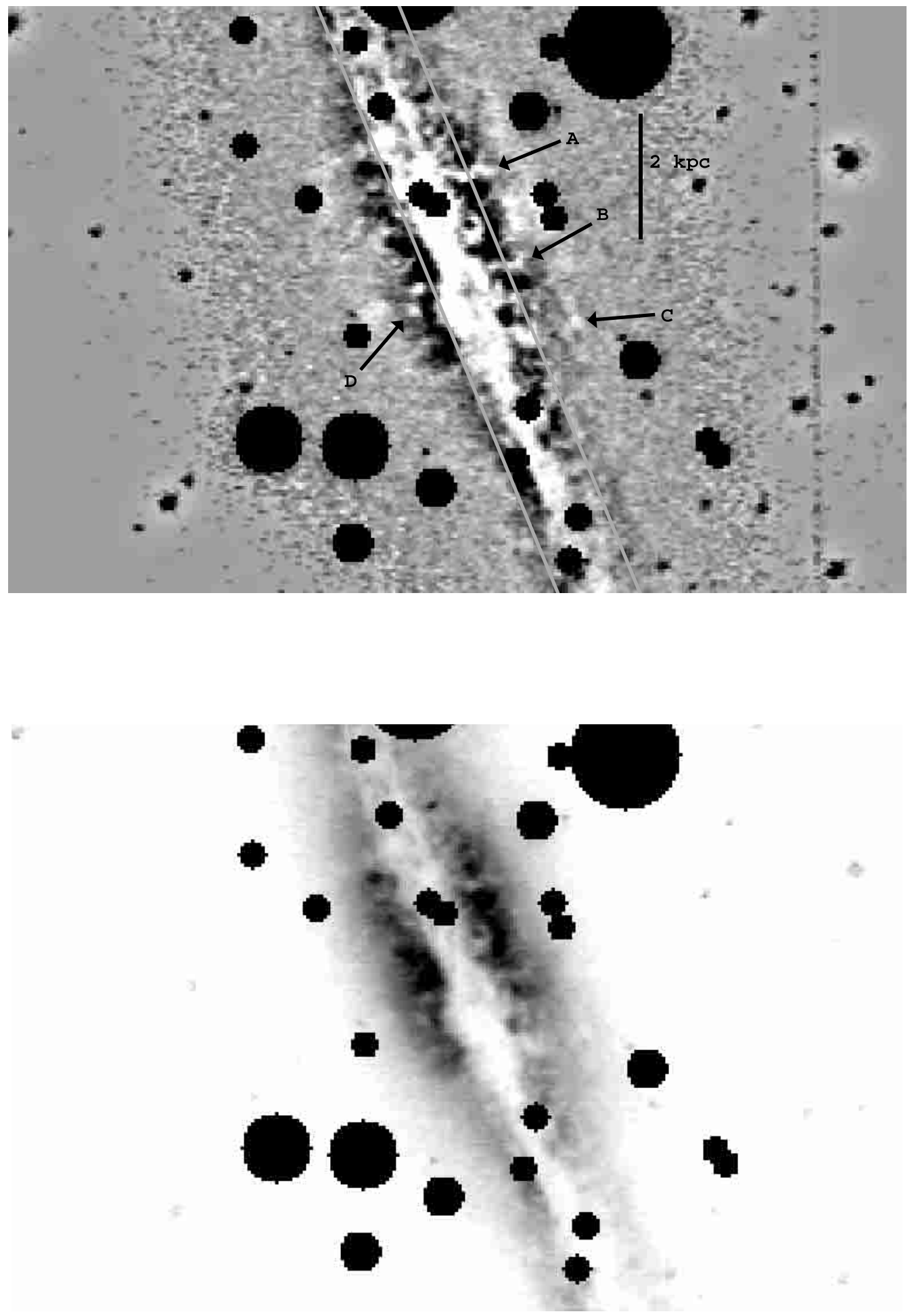

Fig. 1. $B$-band image (bottom) and an unsharp-mask $B$-band image (top) of the central part of NGC 891 shown to the same scale. Arrows indicate examples of dust features considered as extraplanar i.e. residing outside the main absorption layer. "A" and "B" refer to typical chimney-like structure extending away from the midplane, whereas "C" and "D" appear to be isolated dust clouds. It should be noted that all these annotated features are recognizable as regions of attenuation in the $B$-band image. A box has been overlayed in order to discriminate between extraplanar extinction and the conventional absorption layer (see text for details). The orientation adopted for both this figure and all subsequent unsharp-mask images (Figs. 2 to 6) is North at the top and East to the left 


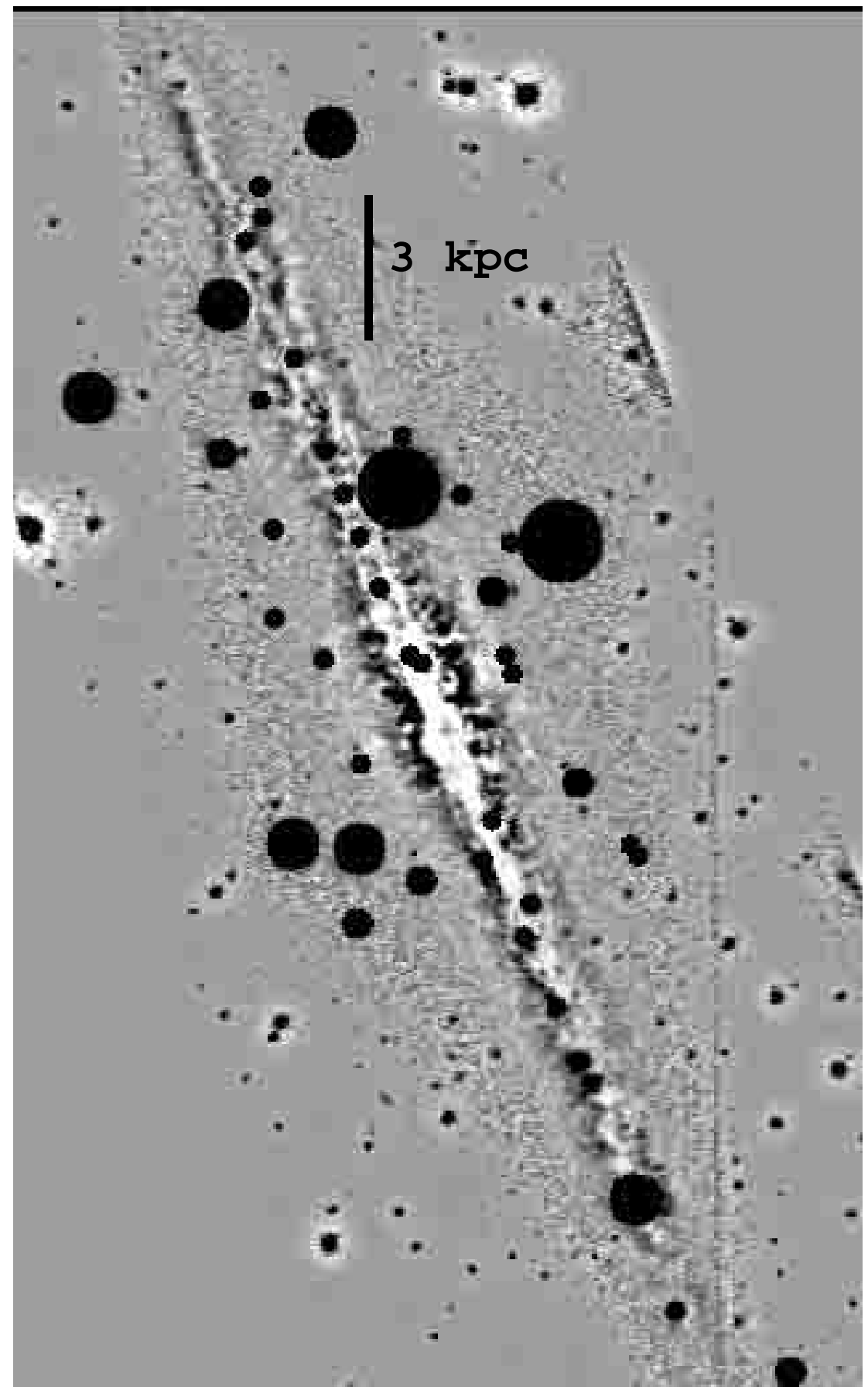

Fig. 2. Unsharp-mask $B$-band image of the whole of the NGC 891 disk 
Table 3. Parametrization of the stellar and absorption disks using radiation transfer modelling. The parameters $z_{\mathrm{d}}$ and $z_{\mathrm{s}}$ represent the exponential scale-heights of the dust and $B$-band stars respectively. Similarly, $h_{\mathrm{d}}$ and $h_{\mathrm{s}}$ denote the exponential scale-lengths of dust and stars, respectively, whilst $i$ corresponds to the inclination inferred for the stellar/dust disk

$\begin{array}{lccccc}\text { Galaxy } & \begin{array}{c}i \\ (\mathrm{deg})\end{array} & \begin{array}{c}z_{\mathrm{d}} \\ (\mathrm{kpc})\end{array} & \begin{array}{c}z_{\mathrm{s}} \\ (\mathrm{kpc})\end{array} & \begin{array}{c}h_{\mathrm{d}} \\ (\mathrm{kpc})\end{array} & \begin{array}{c}h_{\mathrm{s}} \\ (\mathrm{kpc})\end{array} \\ \text { NGC 891 } & 89.8 & 0.31 & 0.43 & 8.0 & 5.7 \\ \text { UGC 4278 } & 87-90 & - & 0.31-0.47 & - & 3.3 \\ \text { NGC 4013 } & 89.9 & 0.13 & 0.19 & 2.6 & 2.6 \\ \text { NGC 4217 } & 85.5 & 0.19 & 0.34 & 4.5 & 3.2 \\ \text { NGC 4302 } & 90.0 & 0.23 & 0.49 & 9.2 & 14 \\ \text { NGC 4762 } & 90.0 & - & 0.43 & - & 4.5 \\ \text { NGC 5023 } & 87.0 & - & 0.17 & - & 2.3 \\ \text { NGC 5746 } & 86.2 & 0.20 & 0.55 & 7.3 & 5.5 \\ \text { NGC 5907 } & 87.0 & 0.13 & 0.34 & 5.3 & 5.0 \\ \text { UGC 10288 } & 87.0 & 0.09 & 0.21 & 5.4 & 3.4\end{array}$

effects will contribute to the apparent height of the main dust lane if the disk is not seen perfectly edge-on.

\subsection{Unsharp-masking}

We adopt an unsharp-masking technique similar to that of previous authors (Sofue et al. 1994; HS99) in locating extraplanar dust features. The $B$-band image is smoothed with a Gaussian function and then the original $B$-band image subtracted from this smoothed version. This accentuates inhomogeneities in the surface photometry such as those caused by dust filaments. Additionally, we experimented with various rectangular median filters with dimensions longer along the in-plane direction (i.e. parallel to the major axis) than the vertical direction. In principle, the unsharp-mask image produced in this way is less susceptible to sharp gradients or cusps in the original surface photometry. However, we did not experience a significant increase in the number of extraplanar dust features that could be detected by this technique. After various trials, we settled on a Gaussian smoothing kernel of $0.5 \mathrm{kpc}$ FWHM which was found to accentuate the chimney structures in an optimal fashion. This represents a compromise scale-length for absorption features which are generally long perpendicular to the disk $(1-2 \mathrm{kpc})$ but short parallel to the disk $(<0.1 \mathrm{kpc})$. Before subjected our images to unsharpmasking we masked out the brightest HII regions within the disk and nay foreground stars projected close to the disk. It is important to point out that the unsharp-mask only served to assist in locating features which could subsequently be recognized as areas of extinction in the original $B$-band image.

Figures 1 to 6 show unsharp-mask images for the 5 galaxies in final sample $\left(i>87^{\circ}\right)$. In Fig. 1 (NGC 891), we highlight various features in the unsharp-mask image

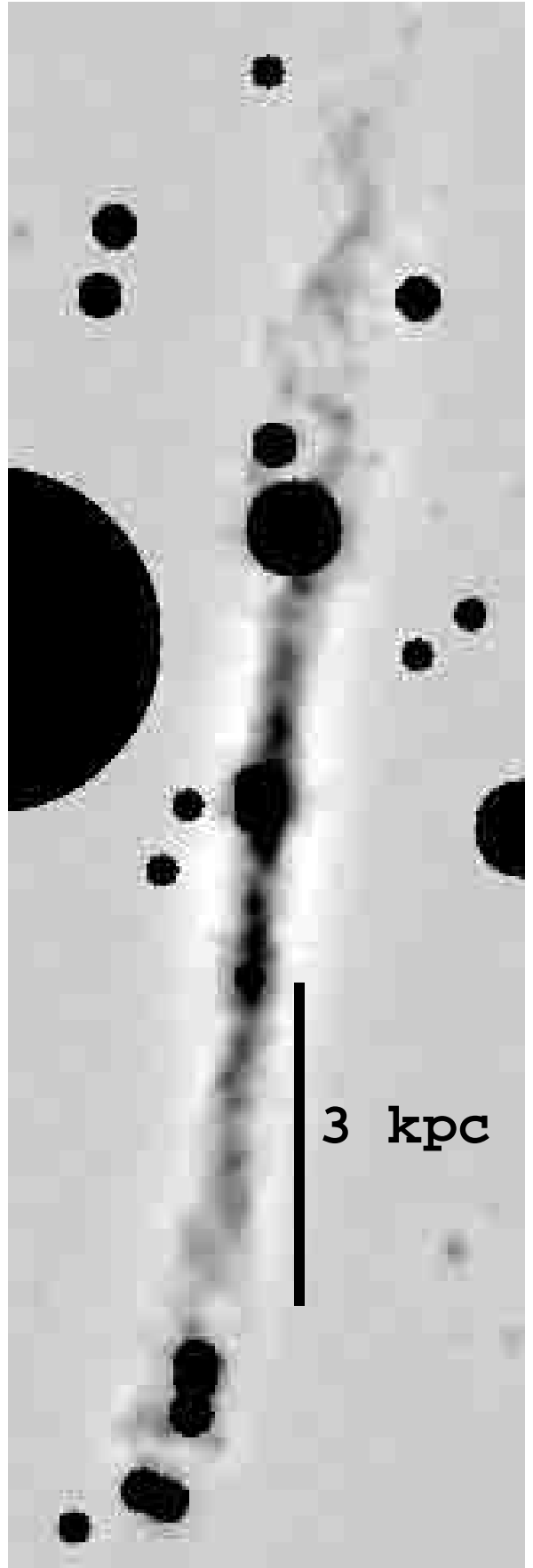

Fig. 3. Unsharp-mask image of UGC 4278 in a blue filter

corresponding to both chimney-like features and isolated high-latitude dust clouds. A rectangular box, of width $4 \times l$, has been superimposed on this figure to show how we have discriminated between "extraplanar" extinction and the main absorption layer (Eq. 1). Absorption features found to stretch from the midplane to regions outside the box were considered as extraplanar, so were isolated dust clouds found at least $2 l$ away from the midplane. 


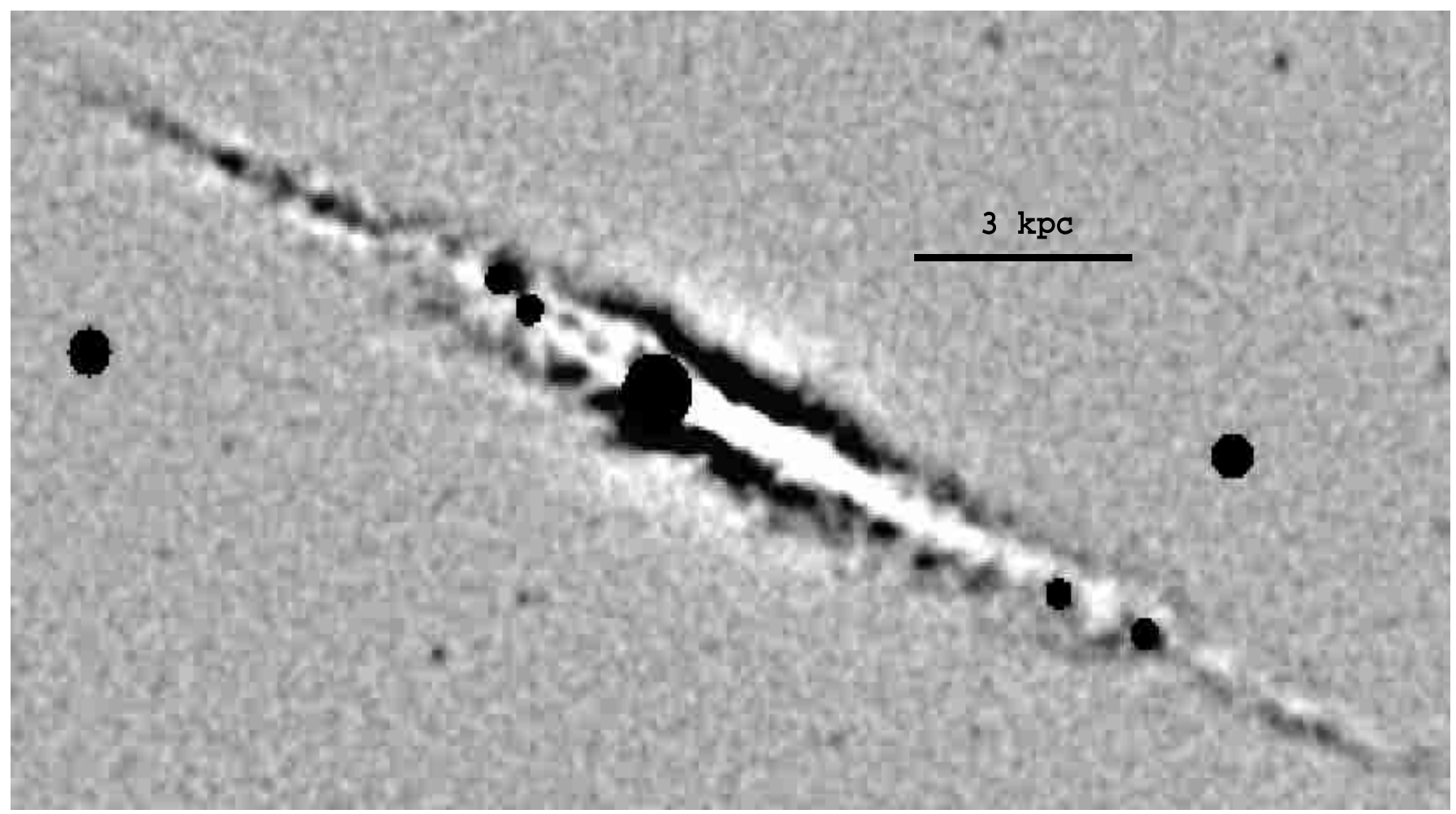

Fig. 4. Unsharp-mask image of NGC 4013 in a blue filter

\subsection{Derivation of opacity}

The opacity of the extraplanar structures was derived in the following way. The relevant features were masked out of the original $B$-band image and then the surrounding pixels used to interpolate the surface brightness over the masked region. By dividing the original surface photometry by the interpolated values it was possible to infer the fraction of light lost due to attenuation by dust. In this way, we derived an opacity $\tau_{B}$ for each of the extraplanar structures. When computing $\tau_{B}$ we integrated the photometry along the whole feature, from one scale-height above the midplane to its maximum height over the disk. When deriving the corresponding dust mass we also used this range in $z$-height.

For a large number of reasons we expect to underestimate the amount of dust in the extraplanar layer. We are probably only sensitive to vertical features on the nearside of the disk and, in our selection of features, we are undoubtedly biased towards dust clouds seen against the luminous backdrop of the bulge. Our unsharp-masking technique is probably completely insensitive to any widely-distributed, diffuse dust that might exist in the lower halo (Davies et al. 1997), requiring as it does strongly-delineated absorption structures that contrast with the unextinguished stellar emissivity. Since we have used a simple, foreground screen approximation to infer $\tau_{B}$, the optical depth we derive for individual features will be severely underestimated. We have attempted to correct for this problem, to first order, by assuming that filaments viewed along lines of sight towards the galaxy centre are in fact situated at radii such that $\frac{1}{4}$ of the stellar light is emitted in front of the feature and passes to the observer without attenuation by the high-lattitude dust. Thus, in this case, we derive a corrected value of $\tau_{B}$ given by:

$$
\frac{I}{I_{0}}=0.25+0.75 \mathrm{e}^{\left(-\tau_{B}\right)}
$$

where $I$ and $I_{0}$ are the observed attenuated and unextinguished light intensities respectively. This correction is roughly consistent with the inference made by HS97, that the reddening exhibited by chimneys viewed towards the centre of NGC 891 is consistent with $0.25 \pm 0.21$ (s.d.) of the light emerging unattenuated. In contrast to Eq. (2), filaments identified at the maximum radius along the major axis are more likely to be situated half-way along the line-of-sight (due to reduced optical depth at the optical edge) and we modify Eq. (2) such that half the stellar light passes through the absorption feature. For intermediate locations along the major axis, $\tau_{B}$ is calculated assuming a linearly-interpolated value between 0.25 and 0.5 for the fraction of light emerging unattenuated. By taking some account of the true star-dust geometry, in this way, we increase the typical value derived for $\tau_{B}$ by $\sim 60 \%$ over the simple screen model. We emphasize that no account has been taken of scattering in this process, nor of dust-clumping in the high- $z$ feature. Both of these would 


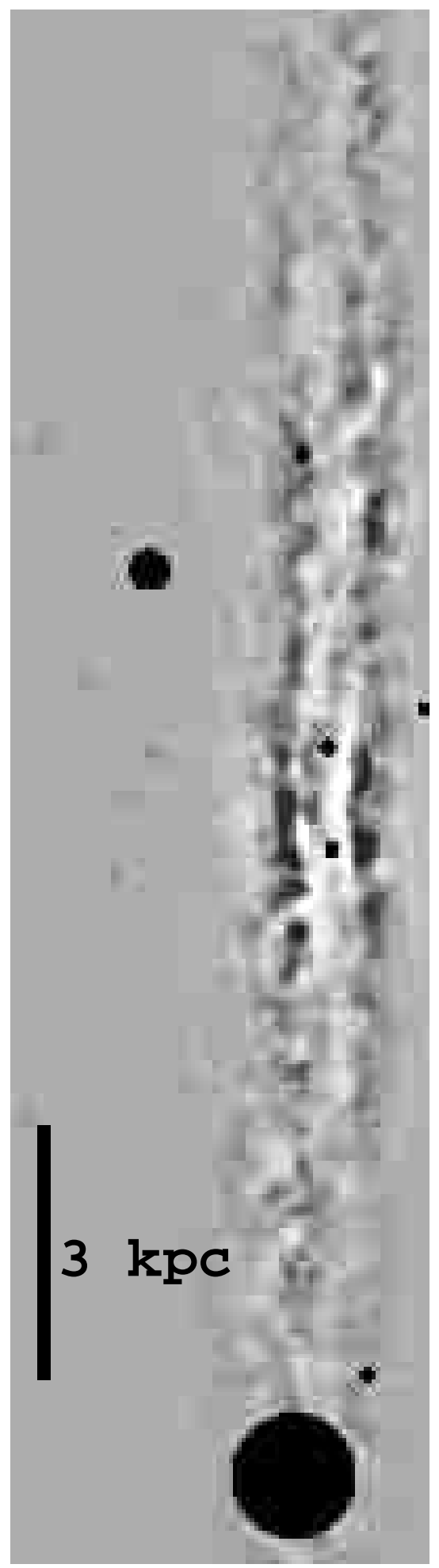

Fig. 5. Unsharp-mask image of NGC 4302 in a blue filter require first-order, upward corrections to $\tau_{B}$ (Witt et al. 1999; Bianchi et al. 1996).

For the $1-2^{\prime \prime}$ seeing conditions characterizing the $B$-band images of our sample (Table 2 ), we expect the point spread function (PSF) to severely "smear out" the chimney features. For example, HS97 estimate from HST images of NGC 891 that the width of many of these structures is only $\sim 30 \mathrm{pc}$ parallel to the disk (equivalent to $\left.0.7^{\prime \prime}\right)$. A consequence of this is that we shall severely underestimate the true value of $\tau_{B}$ (although we expect to recover the total dust mass present by integrating $\tau_{B}$ over the whole of the dust feature). Indeed, a comparison between chimneys classified by HS97 in their Table 3 (under seeing conditions of $0.6^{\prime \prime}$ ) with the same structures identified in our own data, shows that we underestimate $\tau_{B}$ by a factor of at least 5 . In contrast, the masses we derive for these selected features agree to within $15 \%$ with the HS97 values.

\section{Results}

\subsection{Prevalence of dust chimneys}

Our primary objectives in this investigation are to ascertain whether dust chimneys are commonplace within quiescent galaxies and how, if at all, they might relate to recent star-formation within the disk. We begin by addressing the first of these issues.

Unfortunately, there are only 5 galaxies, out of the original sample, which are orientated sufficiently close to edge-on $\left(i>87^{\circ}\right)$ to carry out an unambiguous search for extraplanar structures. This subset is too small to result in any unequivocal statements about the overall occurence of dust chimneys. Of the 5 galaxies depicted in Figs. 1 to 6, 3 harbour very chaotic dust lanes (NGC 891, NGC 4013, NGC 4302) with numerous curvilinear structures extending up to $2 \mathrm{kpc}$ from the midplane. Many of the extinction features extend to a $\mathrm{kpc}$ or so above the midplane before arching back towards the disk. Others show no sign of reconnecting with the main dust layer and appear to fragment at maximum $z$ height. More spherically-shaped dust clouds are also recognizable, usually at $z \simeq 2 \mathrm{kpc}$, and appear completely isolated from the disk. The maximum $z$-height at which extraplanar dust is discernible is about the same in all 3 galaxies $(z=1.7 \mathrm{kpc})$. (However, we might expect that diminishing bulge light would preclude a detection of extinction features at much greater $z$-heights). Of the 2 remaining galaxies in Figs. 1 to 6 , UGC 4278 appears to contain only small amounts of extraplanar dust and NGC 4762 none at all.

In the HS99 study, 5 galaxies are labelled as manifesting chimneys (out of a total of 7 ). Three of these objects appear in our final sample (NGC 891, NGC 4302, NGC 4013) but the other two (NGC 4217 and NGC 5907) 
we have already rejected as not being sufficiently edge-on to make any unambiguous statements about extraplanar dust. It is interesting to note that NGC 4217 (and possibly NGC 5746 from our original sample) both exhibit some filament-like features which extend to larger (apparent) $z$ heights than can probably be accounted for by projection of disk structure onto the plane of the sky. However, in the strictest sense (using the $i>87^{\circ}$ criterion), the HS99 study and the present work indicate collectively that 5 out of 10 spirals possess dust chimneys. Whilst the current statistics are small, we concur with HS99 that the "chaotic" dust lane in NGC 891 can no longer be considered anomalous and, indeed, high-lattitude dust appears to occur in half of all quiescent spiral galaxies.

The detection of structures both below and above the midplane, argues against warps being the cause of the chimney phenomenon. However, it is less easy to dismiss the possibility of flaring in the outer regions of the disk being a contributory factor. Optical images of spirals more inclined to the line-of-sight, such as NGC 253 $\left(i=78^{\circ}\right)$, do exhibit numerous protuberances from the main dust lane and, moreover, at various distances from the nucleus (Sofue et al. 1994). In Fig. 7, we highlight similar features evident in two other slightlyinclined spirals that have recently come to our attention (NGC 2976 and NGC 4928). Although line-of-sight ambiguities would inhibit us from labelling these particular features as definitely extraplanar, their similarity to the vertical structures depicted in Figs. 1 to 6 is striking. The fact that such structures are not concentrated at the edge of the disk, appears to confirm that flaring cannot be the mechanism producing dust chimneys. HS97 have also presented cogent arguments, based on velocity information for the gas in NGC 891 and its relationship to the dust chimneys, as to why gas-flaring cannot be the reason why chimneys are observed in this galaxy.

In Table 4, we present the mean optical depths of extraplanar dust features found in our final galaxy sample. We also include total masses for dust and gas residing outside the main disk. The gas mass is calculated assuming a gas-to-dust ratio of 150 i.e. appropriate to gas with a solartype metal abundance (Whittet 1992). The typical optical depth for the extinction features identified in Figs. 1 to 6 is estimated as $\tau_{B}=0.1-0.2$. Our poor resolution of the features, combined with the neglect of scattering effects, means that we could have underestimated the true column density of dust by at least a factor of 5 . Thus an optical depth closer to unity is probably more likely. The extraplanar gas associated with the chimneys is $\sim 1 \%$ of the total $\mathrm{HI}+\mathrm{H}_{2}$ mass, assuming a canonical mixture of gas and dust in these structures. We note, however, that if grains are expelled from the disk by radiation pressure rather than convective flow (Sect. 6.4), dust and gas might be effectively decoupled from one another. Under such circumstances the amount of gas in the extraplanar layer may be considerably different to our estimate.
HS99 do not appear to list total masses for the extraplanar structures identified in their larger edge-on galaxy survey (although they give masses for individual, selected features). For NGC 891 (HS97), an estimate of $\sim 2 \%$ of the neutral ISM is cited, which is significantly higher than the value of $0.3 \%$ we give in Table 4 . The HS97 estimate relies on 12 well-studied features epitomizing all 120 structure identified in their investigation. Thus some of the discrepancy may arise from an extrapolation of 12 to 120 objects. However, it is also likely that the current work is less sensitive than the HS97 study - our larger PSF means that we ultimately overlook both smaller and less optically-thick high- $z$ clouds. We view the extraplanar masses listed in Table 4 very much as lower limits. In 5.1 we analyse the thermal emission from NGC 891 in the submm regime in order to obtain an upper limit on the amount of dust and gas situated outside the disk.

\subsection{Potential energy of chimneys}

We derived the potential energy of the extraplanar structures, identified in the previous section, using a numerical simulation based on an exponential disk of stars and a spherical dark matter halo. In the first instance we concentrate on NGC 891 for which the vertical features are best resolved. Using the dust free photometry from the Xilouris radiation transfer model, we infer a central luminosity density $\left(\begin{array}{llll}5.5 & 10^{9} & L_{\odot} \mathrm{kpc}^{-3}\end{array}\right)$, a radial scale-length $(3.9 \mathrm{kpc})$ and an exponential scale-height $(0.35 \mathrm{kpc})$ for the stars. The stellar luminosity density is converted to a mass density assuming a mass-to-light ratio of unity. Although the rotation curve of NGC 891 has been investigated quite closely (Bahcall 1983), the dark matter halo can be described by a variety of models and is not well constrained in this case. Thus we refer to a fit by van Albada et al. (1985) to the rotation curve of NGC 3198 - a galaxy similar in type, size and total magnitude to NGC 891. Following their inference of how dark matter scales with luminous matter, the dark matter radial mass density was prescribed as follows:

$\rho(r)=\frac{5.710^{7}}{1+(r / 11.4)^{2.1}}$

where $r$ is the galactic-centric radius, in kpc, and $\rho(r)$ constitutes the mass density in $M_{\odot} \mathrm{kpc}^{-3}$.

A catalogue was created of the 60 or so extraplanar dust features in NGC 891, containing the relevant optical depth, mass, height above the plane and radial distance. We allow, as we did previously, for the likelihood that features seen towards the nucleus are situated on the nearside of the disk. Thus, we set $r=0.5 \times R_{25}$ for features viewed towards the centre, $r=R_{25}$ for structures identified at the maximum distance along the major axis and interpolated values of $r$ for the features between these extremes. The catalogue records are fed into the numerical simulation 


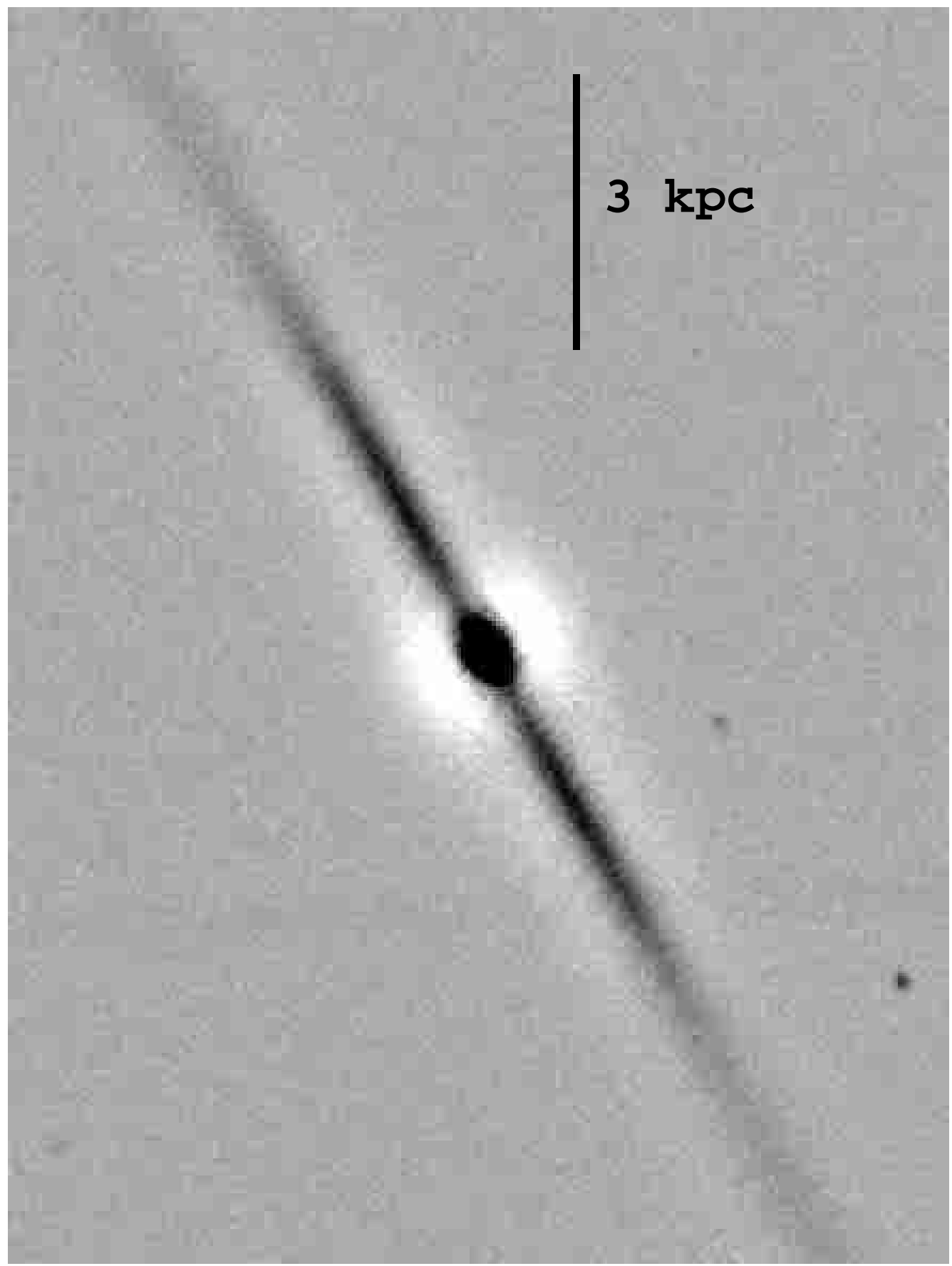

Fig. 6. Unsharp-mask image of NGC 4762 in a blue filter

which calculates, stepwise, the amount of energy that is required to lift any particular feature above the relevant part of the plane. In Table 5, we show entries from the catalogue, including the derived potential energies, for the 4 extraplanar features highlighted in Fig. 1.

We derive a total potential energy of $3.910^{54} \mathrm{ergs}$ for all the extraplanar structures in NGC 891 with a mean value of $6.410^{52}$ ergs per structure. The energies are similar to the values found by HS97 but this is coincidental because these authors use a plane-parallel model with a much lower mass density in the midplane $(0.185$ $\left.10^{9} M_{\odot} \mathrm{kpc}^{-3}\right)$ but a considerably larger stellar scaleheight $(0.7 \mathrm{kpc})$. Given that one of our key objectives will be to relate star-formation in the disk with the occurence of dust chimneys, we compare briefly now the derived potential energy with the anticipated injection of 

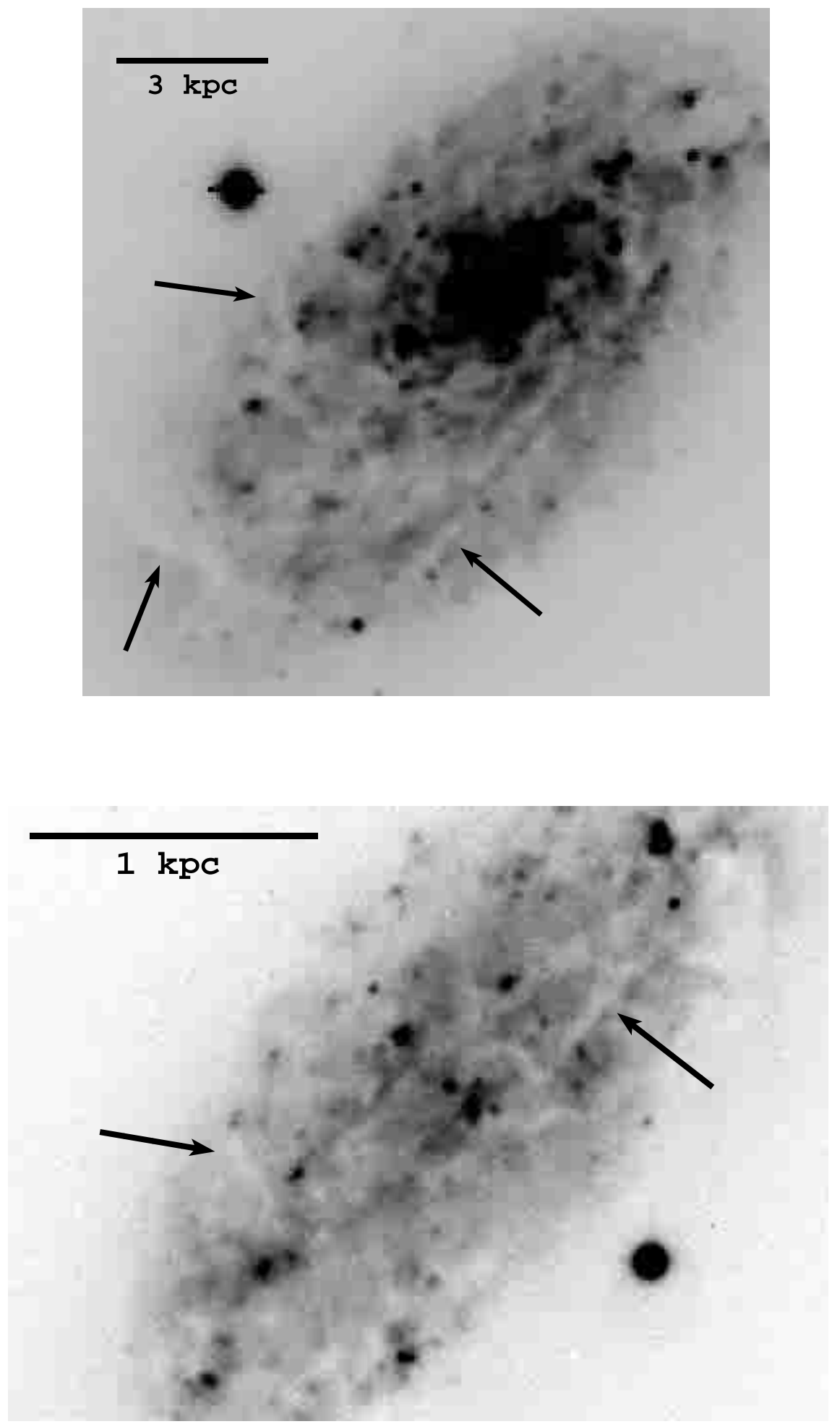

Fig. 7. B-band images of the inclined galaxies NGC 4928 (top) and NGC 2976 (bottom). The arrows indicate possible chimney structures. Such features occur at a range of distances from the nucleus and do not appear to be associated with flaring towards the edge of the gas disk. CCD frames for these objects were obtained from the ING telescope archive (1200 s JKT exposure for NGC 2976 and $1000 \mathrm{~s}$ INT exposure for NGC 4928) 

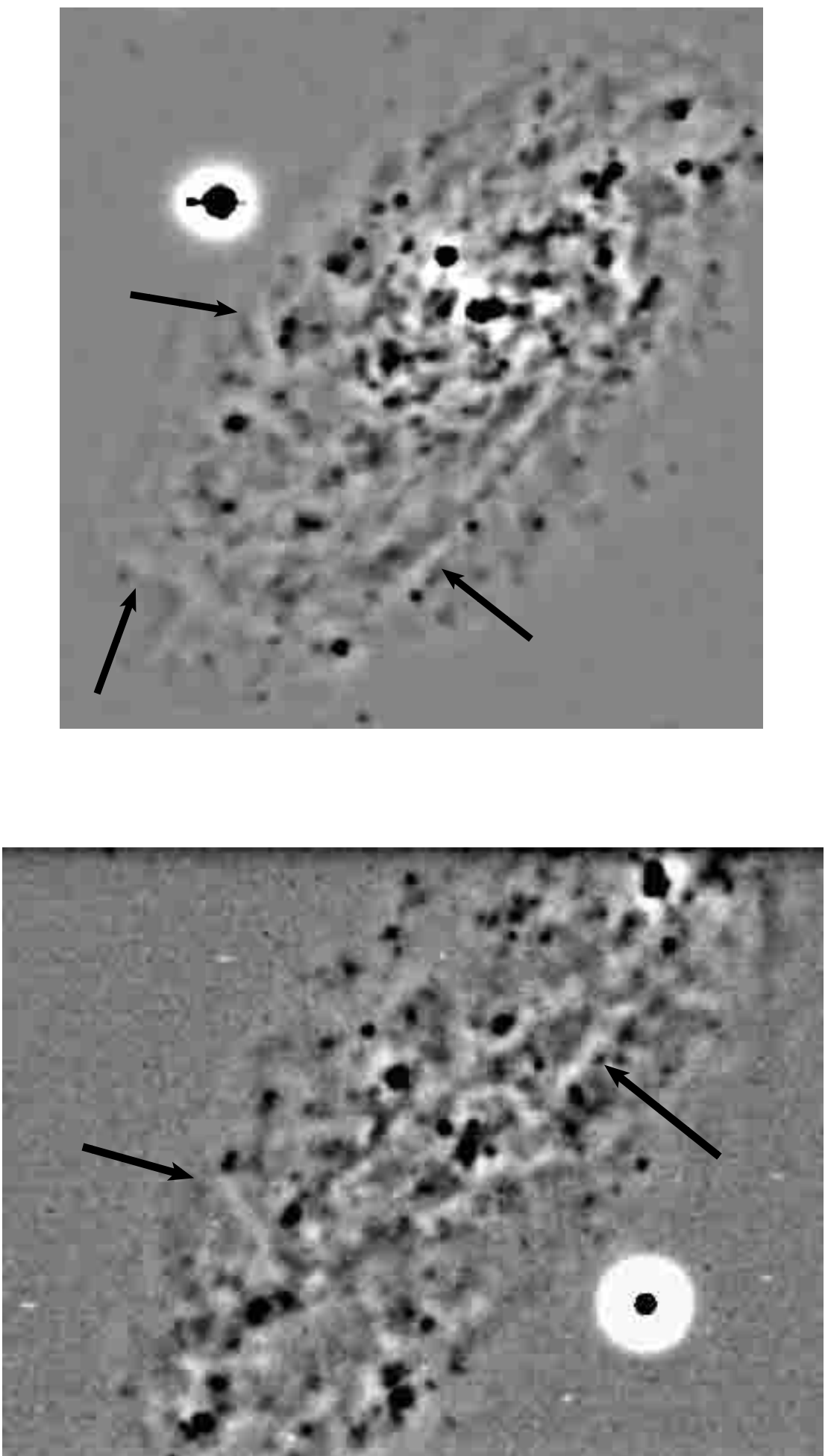

Fig. 8. Unsharp-mask images of the galaxies shown in Fig. 7 
Table 4. Properties of the extraplanar dust structures identified in the final, edge-on sample. $<\tau_{B}>$ is the mean $B$-band opacity of the individual features, whilst $M_{\mathrm{d}}$ and $M_{\mathrm{g}}$ represent the total dust and gas masses found outside the main disk. $M_{\mathrm{d}}$ and $M_{\mathrm{g}}$ have been calculated assuming Galactic-type grains are present according to a solar gas-to-dust ratio. $M_{\mathrm{G}}$ is the total gas mass of the disk $\left(\mathrm{HI}+\mathrm{H}_{2}\right)$. Except for NGC 891, where atomic and molecular gas masses are available in the literature (Scoville et al. 1993), we use $21 \mathrm{~cm}$ line fluxes in the RC3 (de Vaucouleurs et al. 1991) to obtain the HI mass and assume that an equal amount of gas is present in molecular form (Devereux \& Young 1990). $<\tau_{B}>$ severely underestimates the true chimney opacity (probably by a factor of 5) because the width of the features are comparable to the PSF of the observations. The distances listed in Table 1 have been used in order to obtain the dust and gas masses

$\begin{array}{lccccc}\text { Galaxy } & <\tau_{B}> & \begin{array}{c}M_{\mathrm{d}} \\ \left(10^{5} M_{\odot}\right)\end{array} & \begin{array}{c}M_{\mathrm{g}} \\ \left(10^{7} M_{\odot}\right)\end{array} & \begin{array}{c}M_{\mathrm{G}} \\ \left(10^{9} M_{\odot}\right)\end{array} & \begin{array}{c}M_{\mathrm{g}} / M_{\mathrm{G}} \\ (\times 100 \%)\end{array} \\ & & & & & \\ \text { NGC 891 } & 0.16 & 2.1 & 3.1 & 9.8 & 0.3 \\ \text { NGC 4013 } & 0.11 & 0.93 & 1.4 & 4.8 & 0.3 \\ \text { NGC 4302 } & 0.19 & 2.6 & 3.9 & 4.0 & 1.0 \\ \text { UGC 4278 } & 0.071 & 0.047 & 0.071 & 2.8 & 0.025 \\ \text { NGC 4762 } & 0.00 & 0.0 & 0.0 & 0.17 & 0.0\end{array}$

Table 5. An extract from a catalogue of extraplanar dust structures in NGC 891. The features refer to those structures annotated in Fig. 1. We have computed the potential energy of the features using a numerical model to describe the stellar disk and dark matter halo

$\begin{array}{ccccc}\text { Feature } & \begin{array}{c}\text { Mean } z \text {-height } \\ (\mathrm{kpc})\end{array} & \begin{array}{c}\text { Radius } \\ (\mathrm{kpc})\end{array} & \begin{array}{c}\text { Mass } \\ 10^{5} M_{\odot}\end{array} & \begin{array}{c}\text { P.E. } \\ \left(10^{51} \mathrm{ergs}\right)\end{array} \\ \mathrm{A} & 0.85 & 9.6 & 8.2 & 87 \\ \mathrm{~B} & 0.73 & 9.9 & 4.8 & 39 \\ \mathrm{C} & 1.5 & 10.5 & 1.6 & 31 \\ \mathrm{D} & 0.92 & 9.9 & 1.1 & 13\end{array}$

energy is focussed directly into the base of the chimney structure.

\subsection{Comparison with $H \alpha$ images}

Within several nearby, starburst galaxies, such as M 82 , an association has been observed between kpc-scale dust outflows and concentrations of recent star-formation in the central disk. Hot gas, pushed out along the minor axis by massive stars and supernova explosions, generates a diffuse nebula of $\mathrm{H} \alpha$ shells and streamers as it collides with ambient gas in the halo. At the same time, dust and neu-

energy from both supernovae and the HII radiation field. As we show in Sect. 6.4, the timescale for the formation of dust chimneys is at least $510^{6} \mathrm{yr}$. Thus, for a supernova rate of $0.01 \mathrm{yr}^{-1}$ and an injected energy of $10^{51}$ ergs per supernova (Leitherer et al. 1992), only $8 \%$ at most of the supernova energy needs to do work in lifting the chimneys out of the plane. We estimate the power in the HII radiation field from the FIR luminosity, $L_{\mathrm{FIR}}$, which we assume to be reprocessed optical and ultraviolet energy. Devereux \& Young (1993) have shown that $L_{\text {FIR }}$ correlates spatially with $\mathrm{H} \alpha$ emission and sites of massive star formation in nearby disks (although perhaps $50 \%$ may arise from the diffuse interstellar medium in some spiral types; Walterbos \& Greenawalt 1996). Thus, using $L_{\mathrm{FIR}} \sim 10^{10} L_{\odot}$ to gauge the radiation energy impinging on grains situated in HII regions, we estimate that only $0.1 \%$ of this energy must be transferred into lifting dust out of the plane. We have assumed here that the photolevitated grains can successfully transfer their upward momentum to the gas during collisions - a situation which is reached relatively quickly in numerical simulations of radiation pressure (Davies et al. 1998). We can conclude, therefore, that both supernovae and radiation pressure provide viable means for the creation of dust chimneys above spiral disks. As pointed out by the referee, however, supernova explosions provide a more natural explanation for dust chimneys because their tral gas from the disk are entrained at the working surface of the expansion so that a network of optically-thick filaments becomes established around the $\mathrm{H} \alpha$ superbubble (Ichikawa et al. 1994; Phillips 1993). In this section, we search for an analogous effect in quiescent disks. We examine the distribution of $\mathrm{H} \alpha$ emission from our sample and attempt to relate it to the occurence of dust chimneys. R96 has already presented a detailed description of the $\mathrm{H} \alpha$ emission from our edge-on galaxies. In addition to bright emission from HII regions, a layer of diffuse ionized gas (DIG) is found in several of the objects. When present, the DIG often extends to several kpc above the disk and possesses an emission measure, perpendicular to the disk, which is comparable to the Reynolds layer in the Galaxy (Reynolds 1992; Kulkarni \& Heiles 1988). Brightest DIG emission tends to occur above regions undergoing enhanced star-formation but, as we discuss later, the mechanism by which the emission-line nebula becomes established above quiescent disks may differ in some ways from the starburst superwind phenomenon.

To compare the distribution of extraplanar dust with $\mathrm{H} \alpha$ emission from both the disk and the DIG layer, we employ two methods. Firstly, we superimpose the unsharpmask images in Figs. 1 to 6 onto the relevant $\mathrm{H} \alpha$ image so that a simple visual inspection can be made. Secondly, we produce a profile by integrating, in $z$-height, both 
the extraplanar dust mass and the $\mathrm{H} \alpha$ emission at each position along the major axis. An overlay of the unsharpmask image over the emission-line frame reveals no obvious correspondance between either (i) the distribution of extraplanar dust and HII regions in the disk, or (ii) the presence of extraplanar dust and an increased DIG brightness. Figures 9 and 10 illustrate the case for NGC 891 and NGC 4302. We find that $\mathrm{H} \alpha$ emission from the extraplanar layer exhibits a far more diffuse distribution than that of the dust chimneys. Overall, the extraplanar ionized gas is far less likely to form discrete structures which might be matched to individual extinction features. Certainly, no parallel can be found with starburst galaxies where dust appears to collect at the working surface of hot gas expelled from the disk.

Our more quantitative approach, of profiling along the major axis, employs a rectangular mask to separate $\mathrm{H} \alpha$ radiation from the disk with that emitted by the DIG layer. The width of the mask is fixed at $4 \times l$ where:

$l=h_{\mathrm{S}} \operatorname{Cos}(i)+z_{\mathrm{S}}$.

Here, $i, h_{\mathrm{S}}$ and $z_{\mathrm{S}}$ are the inclination, stellar scale-length and stellar scale-height, respectively. These are taken from the radiation transfer parameters given in Table 3 (cf. Eq. (1) used to define the extraplanar layer for the dust). The $\mathrm{H} \alpha$ flux is integrated, in $z$-height, up to the box boundary for emission from the disk and beyond the box boundary for radiation from the DIG. In Fig. 11, we plot the amount of extraplanar dust at each point along the major axis against the corresponding $\mathrm{H} \alpha$ emission from HII regions in the disk. In Fig. 12, the extraplanar dust is compared with the corresponding DIG emission. A sampling interval, along the major axis, of $1 \mathrm{kpc}$ has been used for all the galaxies depicted. Of the 4 galaxies plotted (NGC 4762 shows no evidence of either extraplanar dust or ionized gas and is therefore not included) only NGC 4013 appears to show any correlation between the distribution of extraplanar dust and the brightness of $\mathrm{H} \alpha$ emission. We checked for stronger correlations over smaller and larger scale-sizes but found that the behaviour of the plotted data remained more or less unaltered for different sampling intervals.

\section{Observational selection effects}

It is certainly something of a surprise to find no obvious correlation between dust expelled from the main absorption layer and either HII regions in the disk or enhanced DIG brightness. Even for NGC 4013, which constitutes a possible exception to this statement, only a general association is evident, with chimneys tending to occur over regions of the disk where the DIG is most luminous. There is certainly no detailed correspondance apparent with, for example, individual dust filaments forming a boundary layer to $\mathrm{H} \alpha$ "bubbles" expanding out of the
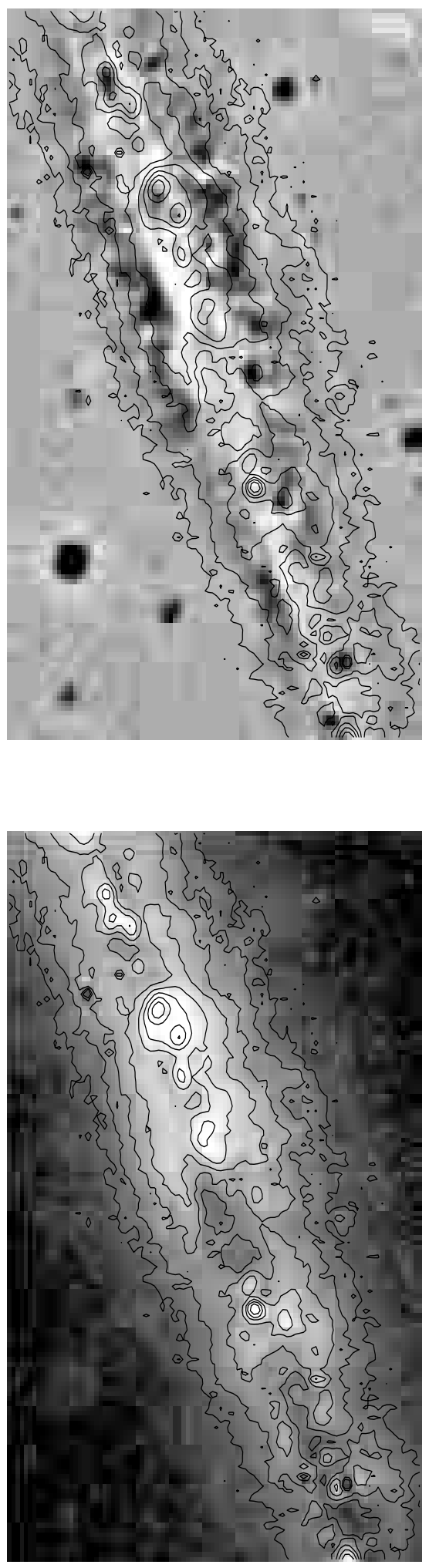

Fig. 9. At the top, $\mathrm{H} \alpha$ emission superimposed as contours onto unsharp-mask $B$-band image of NGC 891. Isophotes begin at $23 \mathrm{EM}$ and increase in steps of $0.5 \mathrm{mag}\left(1 \mathrm{EM}=210^{-18} \mathrm{ergs}\right.$ $\left.\mathrm{cm}^{-2} \mathrm{~s}^{-1}\right)$. The same contours are overlayed onto a greyscale $\mathrm{H} \alpha$ image at the bottom. Both images subtend $2^{\prime} \times 3^{\prime}$ and are orientated with North at the top and East to the left 

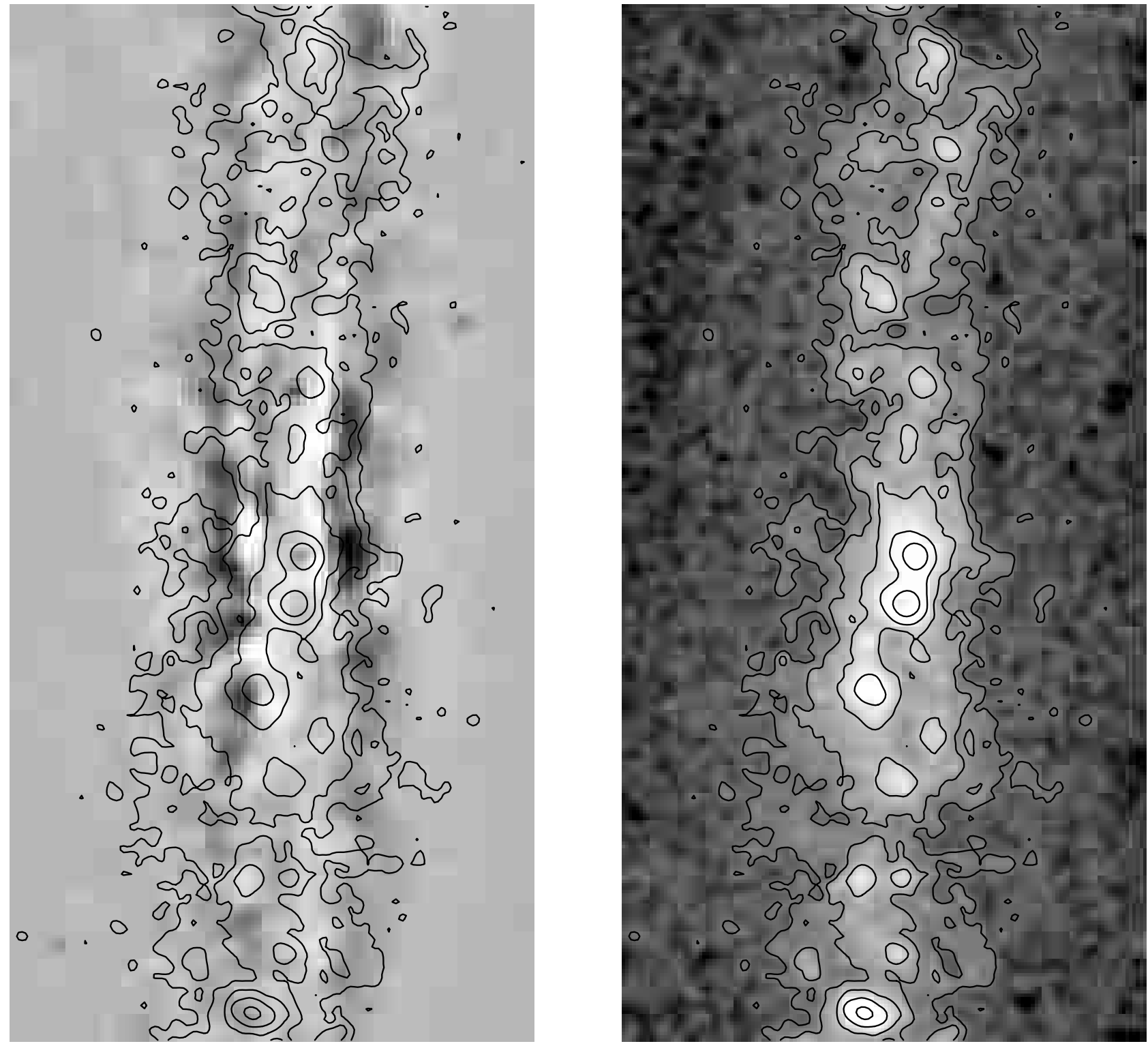

Fig. 10. Left, $\mathrm{H} \alpha$ emission has been superimposed as contours onto unsharp-mask $B$-band image of NGC 4302 . Isophotes begin at $10 \mathrm{EM}$ and increase in steps of $0.75 \mathrm{mag}\left(1 \mathrm{EM}=210^{-18} \mathrm{ergs} \mathrm{cm}^{-2} \mathrm{~s}^{-1}\right)$. The same contours are overlayed onto a greyscale $\mathrm{H} \alpha$ image shown on the right. Both images subtend $0.75^{\prime} \times 1.5^{\prime}$ and are orientated with North at the top and East to the left

disk. Observationally, there are severe obstacles in looking for a correlation between $\mathrm{H} \alpha$ emission and extraplanar dust. As far as the dust is concerned we are most sensitive to dust features on the near-side of the disk (given that we have selected on the basis of optical extinction). At the same time, $\mathrm{H} \alpha$ emission emanating from anywhere up to $z$-heights of $2 \mathrm{kpc}$ is susceptible to extinction from these same features. Indeed, for a typical chimney optical depth of $\tau_{B} \sim 1,6563 \AA$ emission-line radiation emitted by the DIG will suffer up to 0.6 mag of extinction depending on whether the dust feature is situated in front of, or behind, the ionized gas. This value excludes attenuation by the more general dust lane although we expect this effect to be generally small (for example, with an edge- on, midplane optical depth $A_{B}=10, A(\lambda=6563 \AA) \simeq$ $0.1 \mathrm{mag}$ at $1 \mathrm{kpc}$ or 4 scale-heights above the disk). Such considerations might argue that the recognition of extraplanar dust and the detectablility of DIG emission are mutually-exclusive. The problem is distinctly exacerbated by our necessary selection of edge-on disks. Choosing less inclined galaxies would, admittedly, mitigate the effects of extinction, but at the same time, we could never be sure that the structures we discover are extraplanar.

In the following sections, we try to assess how well our unsharp-masking technique reveals the true distribution of extraplanar grains. We do this in two different ways. Firstly, we use images of the (optically-thin) submillimeter emission recently detected from NGC 891. 
Secondly, we examine $B$-band images of the edge-on spiral NGC 55, which at a distance of only $1.6 \mathrm{Mpc}$ offers superior spatial detail over galaxies comprising the R96 sample.

\subsection{Submillimeter images of $N G C 891$}

Alton et al. (1998a) obtained deep 450 and $850 \mu \mathrm{m}$ images of NGC 891 with a spatial resolution of $10^{\prime \prime}$ and $16^{\prime \prime}$ respectively (see also Israel et al. 1998). These submillimeter maps, obtained with the SCUBA array on the James Clerk Maxwell Telescope, trace primarily thermal emission from cold dust residing in the edge-on disk (temperature $\sim 17 \mathrm{~K}$ ). Notably, however, fairly structured submm emission is detected up $z=2 \mathrm{kpc}$ in both these images (Fig. 13). Given a nominal FWHM beamwidth of $15^{\prime \prime}$ $(700 \mathrm{pc})$ and $9^{\prime \prime}(400 \mathrm{pc})$ at 850 and $450 \mu \mathrm{m}$, respectively, it is reasonable to ask whether this apparent high- $z$ emission can identified with the extinction chimneys identified in our $B$-band image. Indeed, an initial inspection of the SCUBA data (Alton et al. 1999b) showed that the extended submm emission is much broader than the instrumental PSF, suggesting that it might well correspond to high-latitude dust. To make a more careful comparison between the extraplanar emission and the vertical extinction features, we use the results of the radiative transfer fit given in Table 3. Adopting the parameters derived for the distribution of optical depth, we produce an image of the standard dust layer which, after smoothing with the appropriate PSF, can be compared with the submm maps. Our objective here is to identify an excess in the submm maps which might be ascribed to the extraplanar extinction features. We utilize calibration images of Uranus, taken at regular intervals during the SCUBA observing schedule, to produce a signal-weighted map of the PSF at both 450 and $850 \mu \mathrm{m}$. Before convolving the model galaxy with the measured PSF we ensure that the chop direction is aligned for both the object and the beam images. This is important because the chop for NGC 891 was along the minor axis and the side lobes of the beam are known to be more pronounced along the chop direction. Having smoothed, in turn, the simulated disk with the respective 450 and $850 \mu \mathrm{m}$ PSF, profiles in $z$-height were created, for both wavelengths, at various locations along the major axis.

It was found that the widths of the simulated profiles were considerably larger than the observed data. After several trials, we established that a modified exponential $z$-height of $0.22 \mathrm{kpc}$ gave a far more consistent fit between model and observation (at both 450 and $850 \mu \mathrm{m}$ and for all locations along the disk). This value is somewhat smaller than the original scale-height of $0.31 \mathrm{kpc}$ but it seems likely that $B$-band radiation transfer modelling of NGC 891 will over-estimate the height of the general dust layer by incorporating high- $z$ extinction features into
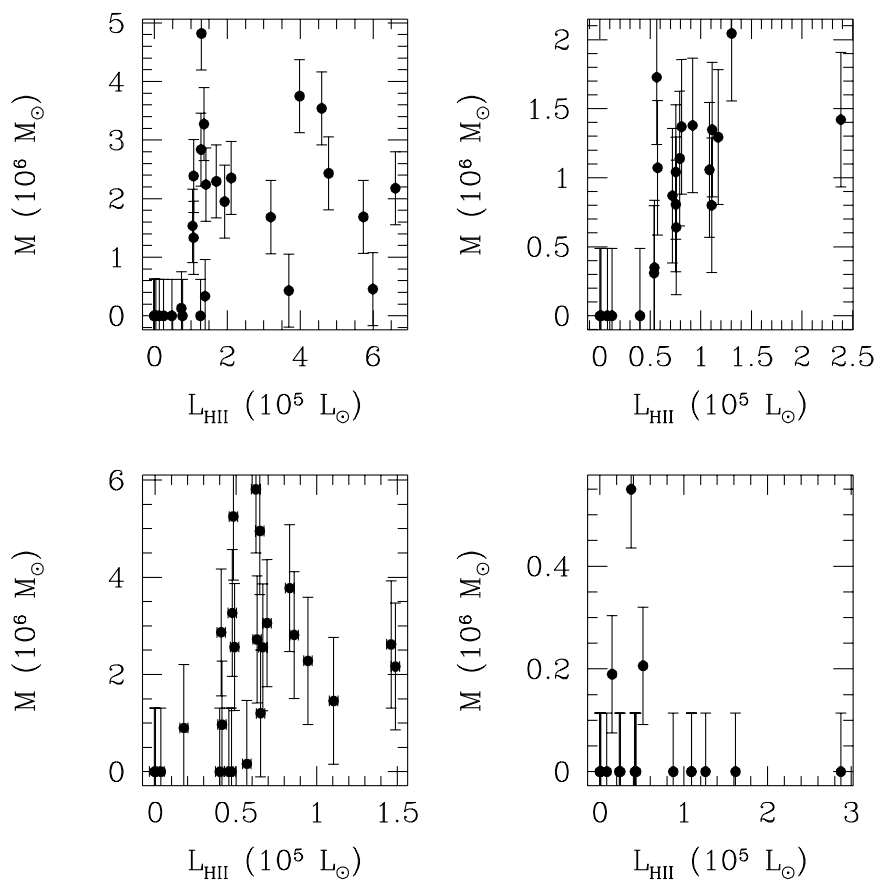

Fig. 11. Extraplanar dust mass plotted against $\mathrm{H} \alpha$ luminosity in the disk. We have integrated the emission line flux and dust mass over $1 \mathrm{kpc}$ intervals along the major axis. The graphs are arranged in the following order: top-left (NGC 891); topright (NGC 4013); bottom-left (NGC 4302); and bottom-right (UGC 4278)
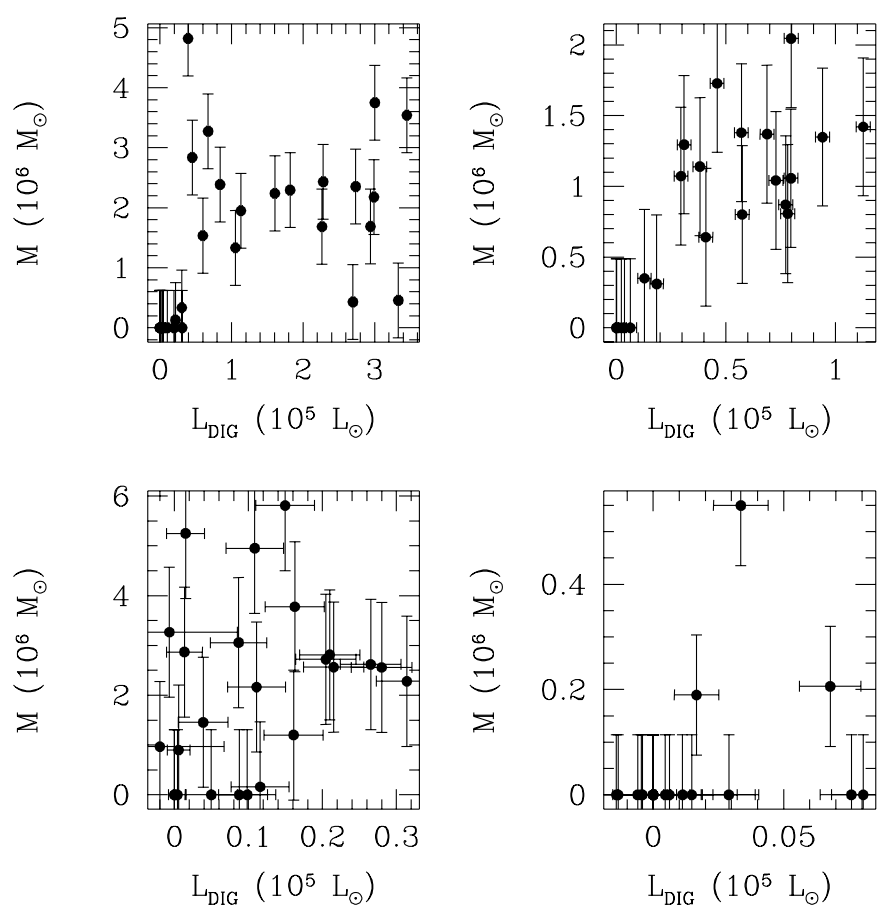

Fig. 12. Extraplanar dust mass plotted against $\mathrm{H} \alpha$ luminosity of the diffuse ionized gas (DIG). We have integrated the emission line flux and dust mass over $1 \mathrm{kpc}$ intervals along the major axis. The graphs are arranged in the following order: top-left (NGC 891); top-right (NGC 4013); bottom-left (NGC 4302); and bottom-right (UGC 4278) 

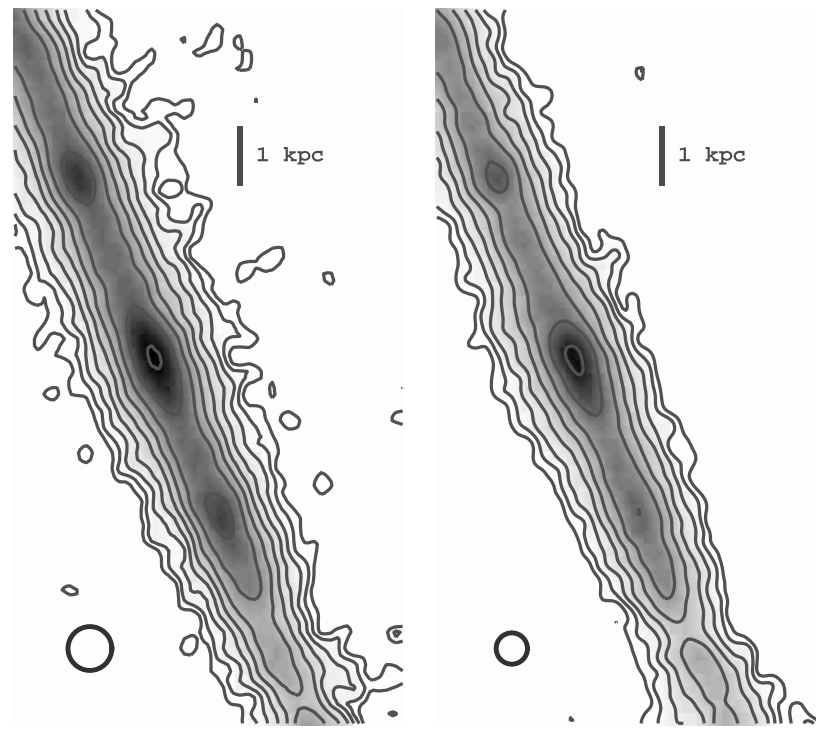

Fig. 13. Submillimeter emission from the disk of NGC 891 . We show the central part of the galaxy at $850 \mu \mathrm{m}$ (left) and $450 \mu \mathrm{m}$ (right) with contours beginning at $3 \sigma\left(10 \mathrm{mJy} / 16^{\prime \prime}\right.$ beam and $40 \mathrm{mJy} / 10^{\prime \prime}$ beam respectively). In each image, the beamsize is represented by a circle at the bottom left and adjacent isophotes are separated by a factor $\sqrt{ } 2(0.4 \mathrm{mag})$. The galaxy is orientated with North at the top and East to the left

the photometric fit (they are particularly conspicuous in a blue filter). Figure 14 shows the simulated profiles, with a $0.22 \mathrm{kpc}$ scale-height, alongside measured profiles of regions of the disk exhibiting high- $z$ structure in the submm image. Our conclusion from this figure is that the detected submm emission even at $30-60^{\prime \prime}(1.5-3.0 \mathrm{kpc})$ is attributable to the main dust layer. The wings of the PSF appear to amplify the tail of the exponential distribution so that spurious high- $z$ "features" are produced which extend up to 3 beamwidths from the midplane. To clarify the situation we also ascertained the fraction of emission occuring at $z>1 \mathrm{kpc}$ in both the 450 and $850 \mu \mathrm{m}$ SCUBA images. This quantity can be expected to vary at different locations along the disk if it is not attributable to the wings of the PSF. Moreover, the high- $z$ flux should correlate at both 450 and $850 \mu \mathrm{m}$ if it has a physical, rather than an instrumental, origin. Figure 15 demonstrates quite clearly that a constant fraction of 450 and $850 \mu \mathrm{m}$ flux is recorded at $z>1 \mathrm{kpc}$ severely undermining our precursory impression that the submm filaments might represent high-latitude dust. The detectability of high- $z$ extinction features in thermal emission is, in fact, doubtful if one considers that a chimney of intrinsic $\tau_{B} \sim 1$ will be smoothed
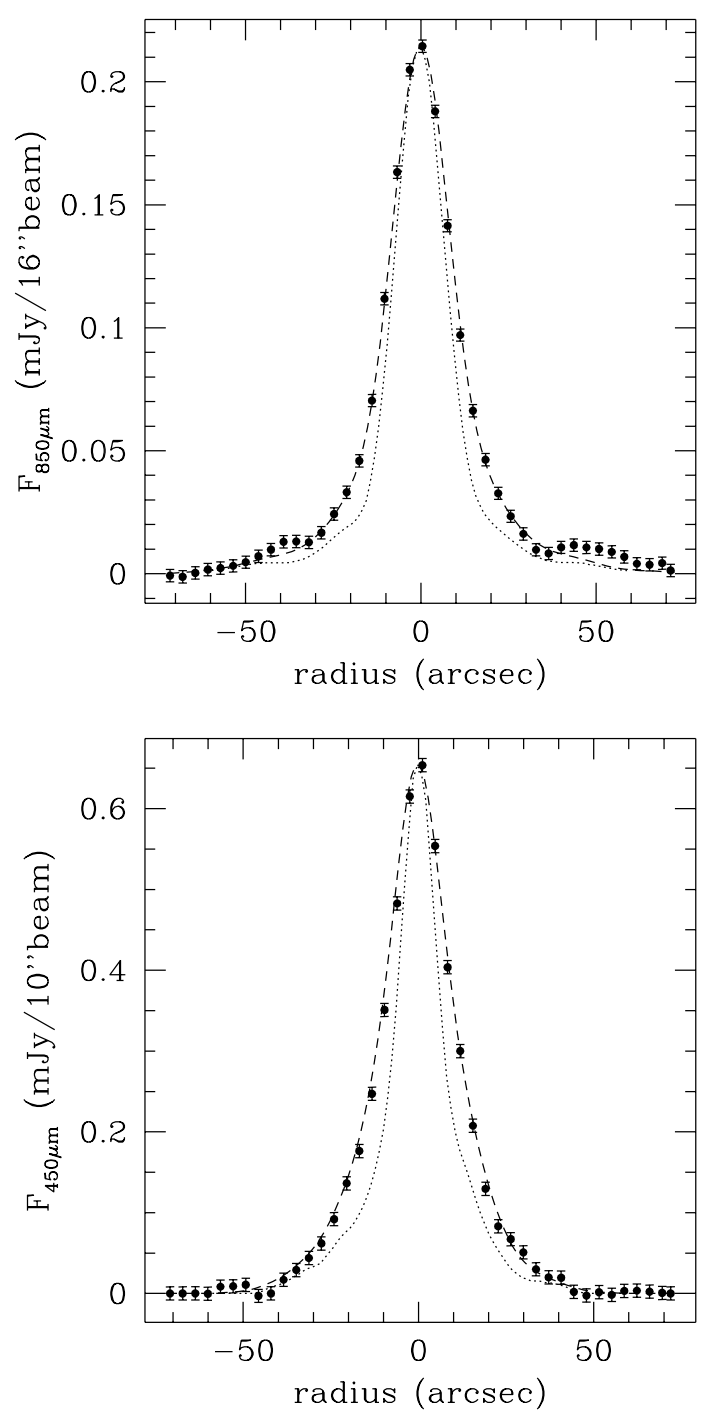

Fig. 14. Profiles at $850 \mu \mathrm{m}$ (top figure) and $450 \mu \mathrm{m}$ (bottom plot) perpendicular to the major axis of NGC 891. In each case, the observed data are denoted by the solid circles and the exponential dust model (e-height $=0.21 \mathrm{kpc}$ ) is represented by a dashed curve. The PSF of the beam, as measured from calibration maps of Uranus, is given by the dotted line

out parallel to the major axis by a $16^{\prime \prime}$ beam in the $850 \mu \mathrm{m}$ image. The $\tau_{B}$ that is then effectively measured is $\sim 0.05$ or $0.5 \mathrm{mJy} /$ beam for cold (17 K) dust (Alton et al. 1999b). The anticipated emission level, therefore, is an order of magnitude smaller than that recorded at $z=1 \mathrm{kpc}$ from the (convolved) main dust layer (Fig. 14).

Although it is disappointing not to be able to use submm images to infer the true distribution of high- $z$ dust, we are still in a position to place an upper limit on the total amount of material in the extraplanar layer. Refering once again to Fig. 14, the observed submm emission seldom deviates more than $2 \sigma$ from the simulated profiles. We therefore attribute, as an upper limit, $2 \sigma$ of the flux to grains located outside the standard exponential layer 


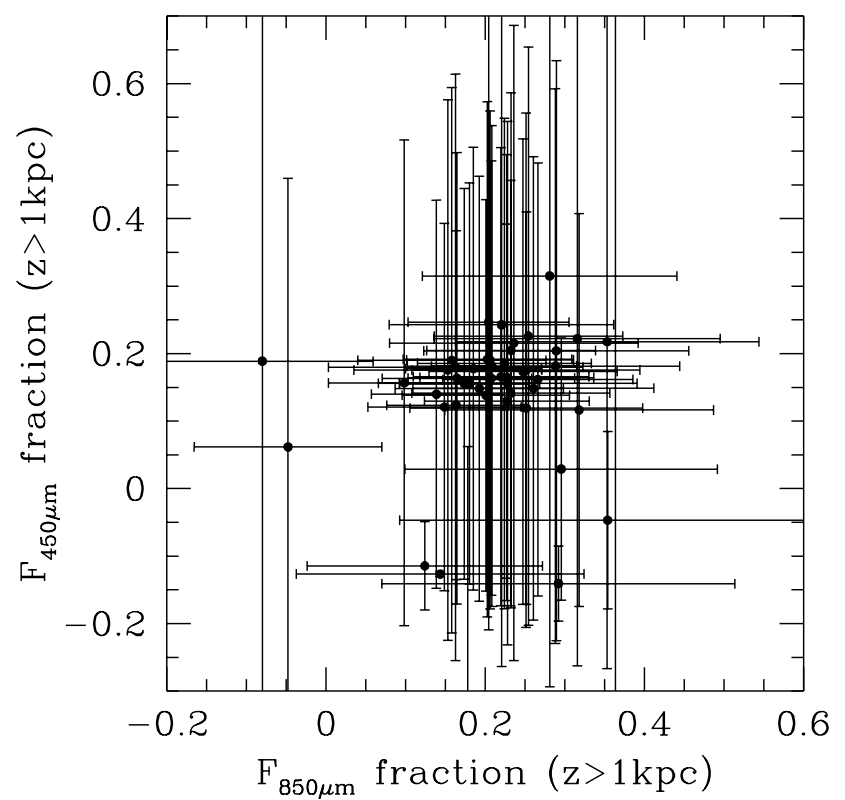

Fig. 15. Submillimeter emission from NGC 891. $F_{450} \mu \mathrm{m}$ and $F_{850 \mu \mathrm{m}}$ represent, respectively, the fraction of 450 and $850 \mu \mathrm{m}$ flux detected at heights greater than $1 \mathrm{kpc}$ above the stellar disk. The data are sampled at $500 \mathrm{pc}$ intervals along the major axis

(5 mJy/16" beam and $16 \mathrm{mJy} / 10^{\prime \prime}$ beam at 850 and $450 \mu \mathrm{m}$ respectively). Integrating over a $z$-height of $1-2$ $\mathrm{kpc}$ we find that $<5 \%$ of both the 450 and $850 \mu \mathrm{m}$ flux can originate from high-latitude dust. Thus, with the proviso that high- $z$ grains are not much colder than $17 \mathrm{~K}$, the SCUBA observations indicate that less than $5 \%$ of galactic dust exists outside the notional absorption layer. For lower grain temperatures this upper limit must be relaxed somewhat - dust temperatures of $10 \mathrm{~K}$ would allow up to $9 \%$ of galactic dust to reside outside the disk. Considered together, however, our optical and submm images for NGC 891 tend to constrain the fraction of dust outside the main extinction lane to $\sim 1-5 \%$. If dust chimneys possess solar-type gas-to-dust ratios, our results imply that only a few percent of the neutral gas present in spiral galaxies reside above the main disk.

\subsection{The nearby edge-on galaxy NGC 55}

At a distance of 1.6 Mpc (Puche et al. 1991), NGC 55 is ten times closer than the galaxies comprising the Rand (1996) sample (Table 1). Under normal conditions of optical seeing, this object provides a remarkable opportunity to resolve regions down to a physical size of $13 \mathrm{pc}-$ a region much smaller than the anticipated width of dust chimneys (30 pc). NGC 55 is a late-type galaxy classified as SB(s)m (de Vaucouleurs et al. 1991). With a $D_{25}$ size of $18 \mathrm{kpc}$, it is physically smaller than most of the spirals in our sample (cf. NGC 891 with a $D_{25}$ corresponding to $37 \mathrm{kpc}$ ) and its overall optical appearance is more irregular than a typical $L_{\star}$ disk. In emission-line radiation, NGC 55 possesses bright, knotted structure in the disk and a spectacular network of curvi-linear filaments extending up to $2.6 \mathrm{kpc}$ away from the midplane (Ferguson et al. 1996; Bomans \& Grebel 1993). The DIG is most conspicuous within the inner $3-4 \mathrm{kpc}$ of the galaxy where the extraplanar $\mathrm{H} \alpha$ structures correspond well to fragmented shells of gas presumably swept up by superbubbles expanding from recent enhanced star-formation in the central disk.

To compare with the $\mathrm{H} \alpha$ image taken by Ferguson et al. (1996), we obtained archival CCD data of NGC 55 in a blue filter. Since this object is located in the Sculptor Group (Dec $=-39^{\circ}$ ) the only image that could be retrieved was a 120 second exposure of the central $9^{\prime} \times 9^{\prime}$ region, which had been taken on the 4-m Anglo-Australian Telescope. These archival frames were reduced in the standard manner and an unsharp-mask $B$-band image created using the same method adopted for the R96 sample. Figure 16 compares the extinction evident in the unsharpmask image with the pronounced network of $\mathrm{H} \alpha$ filaments located at the centre of the galaxy. We have superimposed on the disk a box to denote the apparent centroid of the most prominent $\mathrm{H} \alpha$ shells. The dust does not extend to such large distances from the midplane as the diffuse emission-line gas (only up to $z=700-1000 \mathrm{kpc}$ in fact). However, there is a suggestion that, perhaps, at either side of the active centre, the dust clouds have been prised away from the midplane by the superbubble's impact on the main gas layer. If this is the case, the situation would be reminiscient of M 82, where the central starburst cavity levers ambient dust and gas away from a rather chaoticlooking absorption layer (Ichikawa et al. 1994).

We should point out that the dust lane in NGC 55 assumes an equally non-uniform, distorted shape in the outlying parts of the disk as it does close to the central star-forming activity. This can be inferred from the extensive $R$-band image of Ferguson et al. which was used in the subtraction of the continuum from their $\mathrm{H} \alpha$ filter. Indeed, the maximum $z$-height at which dust manifests itself through $R$-band absorption varies little along the major axis and is typically no more than $\sim 1 \mathrm{kpc}$. Parallel to the disk, the horizontal width of the "vertical" absorption structures is generally $\geq 40 \mathrm{pc}$ which is consistent with the width of dust chimneys in NGC 891, as measured by the Hubble Space Telescope (HS97). The fact that vertically-extending dust structures do not appear to be concentrated where the DIG is brightest might be considered indicative that disruption in the disk and halo occur on different timescales. A chaotic 
dust lane may be the remnant of a more widespread epoch of star-formation whilst the brightest $\mathrm{H} \alpha$ filaments above the central disk represent a more recent episode of stellar creation. We elaborate these ideas in the next section.

Although we obtain significantly more spatial detail in using NGC 55, our reliance on unsharp-masking to identify concentrations of high-latitude dust means that we are always sensitive to the relative geometry between stars and dust. The proximity of NGC 55, however, makes it an ideal candidate for future submm/mm imaging where sampling of the optically-thin thermal emission from high$z$ grains can be carried out.

\section{Discussion}

\subsection{Relating high-z dust to the DIG}

It is somewhat surprising not to find an association between extraplanar dust and increased $\mathrm{H} \alpha$ emission in either the disk or DIG layer. As mentioned previously, the observational methods we employ to map out the respective distributions of extraplanar dust and ionized gas have serious limitations. Nonetheless, we might expect to see some indication in our optical and emission-line images of NGC 55 that dust chimneys either form close to HII regions or are entrained by the gas they expel from the disk. In FIR-bright, starburst galaxies, where the dust chimneys and DIG appear well coupled, the $\mathrm{H} \alpha$ radiation originates as a shock cooling-line as the superbubble expands into the ambient halo gas (Heckman et al. 1990). The source of the DIG in quiescent spirals is more ambiguous. Energetically, it appears that only photons from massive stars in the disk below are capable of maintaining ionization of the DIG layer (Kulkarni \& Heiles 1988). What is not clear is whether some sort of initial expulsion mechanism, akin to that operating in starburst galaxies (SNe explosions and fast stellar winds), is required for gas to reach its present location $1-2 \mathrm{kpc}$ above the midplane. Such a process, if present, must evacuate a hole in the main gas layer with the possibility that dust is lifted out of the plane at the same time. Indeed, if high-energy photons from HII regions are expected to reach $z$-heights of a few kpc (where they can ionize the DIG), a significant proportion of the dust directly "above" and surrounding the star-forming site must be swept clean from the optical path. The dimensions of the emission-line shells and bubbles in NGC 55 suggest than an area of $0.7 \times 0.7 \mathrm{kpc}^{2}$ must be cleared from the central disk of NGC 55 in order for $\lambda<900 \AA$ photons to reach the DIG unimpeded (Fig. 16). For a face-on optical depth of $\tau_{B} \sim 1$ through the centre of the galaxy (Xilouris et al. 1999), $\sim 10^{5} M_{\odot}$ of dust must then have been expelled from this region assuming Galactic type grains are present (Whittet 1992). Comparing with Table 4, this mass of material is suffi- cient to create numerous dust chimneys if the appropriate chimney formation mechanisms are present.

It is almost certain that the material visible as extraplanar dust in our $B$-band images represents a very different phase to the emission-line gas traceable at $6563 \AA$. If Galactic-type gas-to-dust ratios prevail, a vertical absorption feature of $\tau_{B} \sim 1$ is likely to be associated with a gas column densities of $\sim 10^{21} \mathrm{~cm}^{-2}$ (Bohlin et al. 1978). For typical chimney dimensions of $30 \mathrm{pc} \times 1 \mathrm{kpc}$ (Sect. 3.2 ) this yields a gas density of $\sim 10 \mathrm{~cm}^{-3}-$ a value well in excess of the density generally attributed to the DIG layer $\left(<0.1 \mathrm{~cm}^{-3} ; \mathrm{R} 96\right)$. This difference in density might explain the difficulty in comparing the respective distributions of the two media. The emission-line gas appears to be considerably more diffuse than that of the extraplanar dust and tends to occupy a far greater filling factor above the disk. We recognize, however, that our unsharp-mask technique is probably insensitive to any diffuse component of dust residing above the disk (material which may indeed relate to the DIG). In general the extraplanar $\mathrm{H} \alpha$ layer in our quiescent galaxies is far more widespread, and much less discernible as individual shell or bubble features, than say the emission-line nebulae characterizing starburst galaxies. The only exception to this might be NGC 891 but, even here, only a small fraction of the DIG appears to reside in well-defined filaments emanating from individual HII regions. The relatively diffuse nature of the emission-line gas in non-starburst systems tends to reinforce the idea that ionization, in this case, occurs via energetic photons escaping from the disk rather than a localized shock due to outflowing gas.

At this point we mention the "thick disk" in NGC 891. A range of gas phases are known to extend to considerable distances above the conventional thin disk in this well-studied system. These different media include X-ray and synchrotron emitting gas as well as the more familiar atomic and molecular components. Table 6 presents the salient properties of each of these extraplanar layers, comparing the scale-heights and densities in each phase. Any robust theory addressing the exchange of material between disk and halo will need to account for the occurence of all these different media. Notably, the dust chimneys in NGC 891 do not appear to correspond closely to any of the other described phases (either in total mass or density). If the vertically-extended $21 \mathrm{~cm}$ emission were shown to be composed of individual, discrete structures, rather than a homogeneous layer, then the gas density of the HI thick disk may be comparable to that of the chimney structures. However, the mass contained in the extraplanar HI gas appears to be an order of magnitude greater than the total chimney mass. In Sect. 6.3, we compare observations of a thick disk in the Milky Way with the distribution of high latitude dust detected at FIR wavelengths (IRAS and COBE data). 

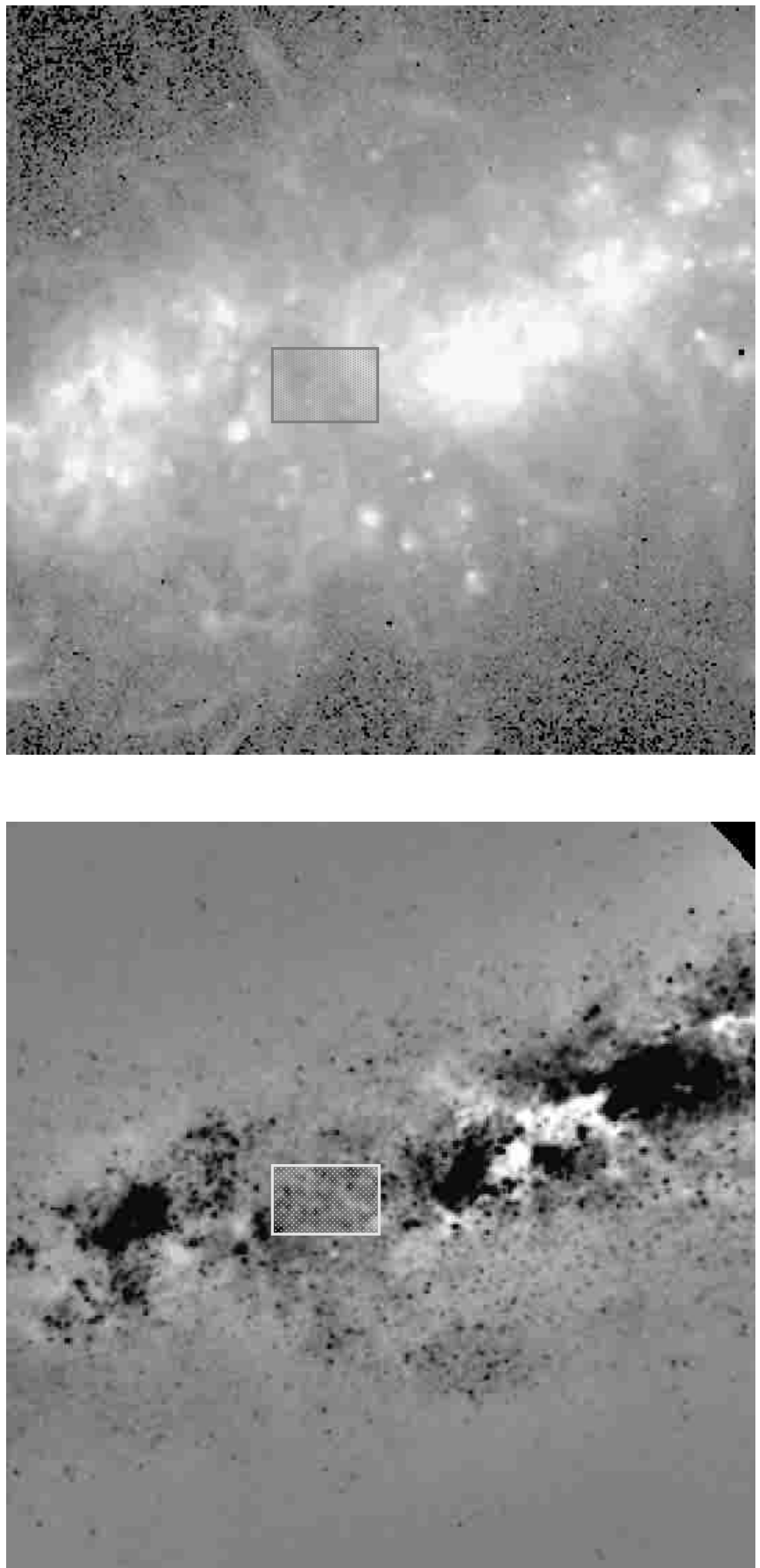

Fig. 16. Images of the inner disk of NGC $55\left(6^{\prime} \times 6^{\prime}\right.$ or $\left.2.8 \mathrm{kpc} \times 2.8 \mathrm{kpc}\right)$. At the top, we show part of the deep emission-line exposure $(\mathrm{H} \alpha+\mathrm{NII})$ obtained by Ferguson et al. (1996). The same area is shown as an unsharp-mask $B$-band image at the bottom. In both cases, a box marks the centroid of the most extensive, high- $z \mathrm{H} \alpha$ filaments 
Table 6. Properties of the "thick" disk in NGC 891. We have collated, from the literature, references to an extended vertical distribution of material. The definition of scale-height depends on the source of the data. We use HWHM to denote the half-width half-maximum and "exp." to represent exponential scale-height (for dust chimneys we give the typical maximum height). The tabulated density refers to the number of atoms per cubic $\mathrm{cm}$ at approximately 1 scale-height above the midplane. The value given for HI is probably a lower limit because a filling factor of unity has been assumed when converting the column density to atoms $/ \mathrm{cm}^{-3}$

\begin{tabular}{|c|c|c|c|c|c|}
\hline Tracer & $\begin{array}{l}\text { Scale-height } \\
\quad(\mathrm{kpc})\end{array}$ & $\begin{array}{c}\text { Density } \\
\left(\text { atoms } / \mathrm{cm}^{3}\right)\end{array}$ & $\begin{array}{c}\text { Temperature } \\
(\mathrm{K})\end{array}$ & $\begin{array}{c}\text { Mass } \\
M_{\odot}\end{array}$ & Reference \\
\hline${ }^{12} \mathrm{CO}$ & 1.5 (HWHM) & 100 & $\sim 20$ & $510^{8}$ & Garcia-Burillo et al. (1992) \\
\hline X-ray & 2.4 (exp.) & $\sim 10^{-3}$ & $410^{6}$ & $\sim 10^{8}$ & Bregman \& Pildis (1997) \\
\hline radio cont. & $1-2($ HWHM $)$ & $?$ & non-thermal & $?$ & Allen et al. (1978); Rupen (1991) \\
\hline $\mathrm{H} \alpha$ & $0.5-1.0$ (exp.) & $\sim 0.1$ & $\sim 10^{4}$ & $\sim 10^{7}$ & Rand et al. (1990); Dettmar (1990) \\
\hline $21 \mathrm{~cm}$ & 1.9 (HWHM) & 0.05 & $\sim 80$ & $2-610^{8}$ & Sancisi \& Allen (1979); Swaters et al. (1997) \\
\hline Dust Chimneys & 1.5 & $\sim 10$ & $?$ & $\sim 10^{7}$ & this work; HS97 \\
\hline
\end{tabular}

\subsection{Age-dependent effects}

One possible explanation for the difficulty in establishing a close spatial correspondance between high-latitude dust and enhanced $\mathrm{H} \alpha$ emission is that the two phenomena might occur during different epochs of the star-formation process. $\mathrm{H} \alpha$ emission, emanating from HII regions, is diagnostic of O-stars and these massive stars are known to possess a lifetime of $\sim 10^{6}$ yr (Leitherer \& Heckman 1995). Thus, if some (small) quantities of gas are already present at high latitudes, ionization of the DIG may proceed relatively promptly after the onset of star formation. $\mathrm{H} \alpha$ images of blue compact dwarf galaxies (BCDs) indicate that the DIG may indeed form early on. Marlowe et al. (1995) detect kpc-scale, diffuse emission-line nebulae in several BCDs known to contain large numbers of WolfRayet stars (the Wolf-Rayet signature indicates a burst age of only a few $\times 10^{6} \mathrm{yr}$; Vacca \& Conti 1992). In contrast, as we show later (Sect. 6.4), the time required for dust chimneys to form is $\sim 10^{7} \mathrm{yr}$. Indeed this appears to be true regardless of whether dust expulsion proceeds by gas convection or radiation pressure. Thus the creation of dust chimneys is synchronized with the emergence of $B$ stars in the star-forming region (few $\times 10^{7} \mathrm{yr}$ ) rather the O-star dominated phase (few $\times 10^{6} \mathrm{yr}$ ).

The scenario outlined above is not without problems. Although the density of atomic gas at heights of $1-$ $2 \mathrm{kpc}$ above spiral disks may be sufficient to allow the DIG to form without prior expulsion of copious amounts of disk gas (Table 6), a path must be cleared if Lyman continuum disk photons are expected to keep the DIG ionized. This pre-condition has already been alluded to in connection with NGC 55 where it was noted that large amounts of dust would have to swept clear of the optical path (enough to produce $\sim 10$ chimneys). Thus, it is not immediately obvious that the DIG can form relatively early on in the star-formation process $\left(\sim 10^{6} \mathrm{yr}\right)$ with chimneys only appearing later $\left(\sim 10^{7} \mathrm{yr}\right)$. Another complication in distilling, temporally, dust chimneys from DIG formation, is that new stars in any particular part of the disk may not

appear in a well-concerted burst but rather over a more protracted time period via sequential propogation (Kunth et al. 1988; Gerola et al. 1980). Thus, at any given time, young stellar clusters of $\sim 10^{7}$ yr may reside in close proximity to concentrations of much younger stars $\left(\sim 10^{6} \mathrm{yr}\right)$. HS99 argue, on the basis of thermal crossing time, that chimneys could remain intact for $10^{7-8}$ years even if they are not confined by magnetic fields.

If the formation of dust chimneys is disjunct from the appearance of the DIG in time rather than in location, it should still be possible to establish a global correlation between the two phenonemona. Thus those quiescent disks which exhibit an enhanced overall DIG brightness might conceivably possess the greatest number of dust chimneys across the whole disk. R96 has shown that the brightest DIG tends to occur above parts of the disk containing a larger number of HII regions. Similarly, Fig. 17 demonstrates that the total DIG luminosity correlates well with FIR luminosity of the disk (where the latter might be treated as a moderately sound indicator of massive star-formation). Equally, we see from the same figure that, those galaxies in our final sample which show the greatest evidence for dust chimneys (NGC 891, NGC 4013 and NGC 4302), do possess the highest FIR and $\mathrm{H} \alpha$ DIG luminosities. Thus there is some suggestion here that more prolific star-formation may lead to increased amounts of extraplanar dust. We emphasize, once again however, that the small number of galaxies composing our final sample prevents us from drawing any unequivocal conclusions here. A larger number of very edge-on galaxies will have to be observed (under very favourable seeing conditions) in order to confirm the trend of increased extraplanar dust with enhanced global star-forming activity.

A deeper understanding of how dust expulsion relates to the star-formation process might be gleaned from suitable observations of the very closest galaxies. Both the LMC and SMC provide detail-rich testing grounds for theories of how young stars might interact with a dusty interstellar medium (ISM). Both galaxies are known to contain a "lacework" of $\mathrm{H} \alpha$ shells and superbubbles 


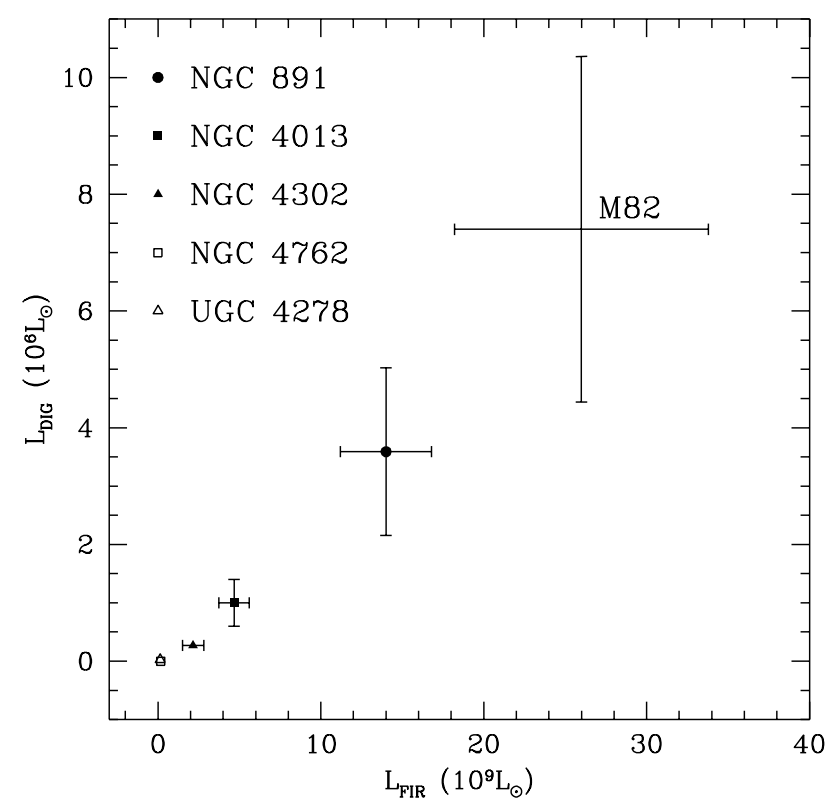

Fig. 17. The luminosity of the diffuse ionized gas (DIG) in the $\mathrm{H} \alpha$ emission-line plotted against the FIR luminosity given in Table 1 . We use $L_{\mathrm{FIR}}$ as an indicator of recent starformation because it is optically thin (any optical tracers of star-formation in the disk will be severely distorted by extinction). We mark the position of the starburst galaxy M 82 on the plot refering to data given by McCarthy et al. (1987)

resulting from recent star-formation across a large part of the ISM (Hunter et al. 1993; Meaburn 1980). A considerable limitation, however, is that neither object is characterized by either a well-defined stellar disk or a particularly dust-rich ISM. The Milky Way might also hold clues as to how recent star-formation and dust chimneys are related, if it can be shown that some fraction of Galactic dust is in fact extraplanar. In particular, the distribution and velocity information of neutral and ionized gas in the local ISM should, in principle, address an intractable problem from another perspective. Accordingly, we now turn our attention to allusions to a "thick disk" in the Galaxy in order to establish whether significant dust resides outside the main gas layer.

\subsection{High lattitude dust in the Milky Way}

The Milky Way has often been cited as being similar to NGC 891, particularly in terms of its velocity field and the distribution of spiral arms (Guelin et al. 1993; GarciaBurillo et al. 1992; Scoville et al. 1993). It is unclear, however, whether our own galaxy contains a thick disk or, indeed, such an active halo as NGC 891. Heiles (1984) famously described a network of "worms" and "shells" which partly comprise the HI disk in the Milky Way. These features can be interpreted as the walls of bubble-like cavities growing in the main gas layer. Although a certain fraction of these structures, the so-called "supershells", possess dimensions comparable to the chimneys identified in our extragalactic sample $(500-1500 \mathrm{pc})$, the majority appear to be only $30-100$ pc in size (Heiles 1984; Hu 1981). A good example of these more diminutive features is the North Polar Spur, which due to its proximity appears conspicuous on HI and cirrus maps of the Galactic plane, but, in fact, has a $z$-height of only 120 pc (Crutcher 1982; Heiles et al. 1980). Curiously, most shells in the Galaxy do not manifest an obvious association with $\mathrm{HII}$ regions or $\mathrm{SNe}$ remnants. Generally, there has been no proven link between shells and any other phenomenon within the disk (although see the more recent radio recombination-line study of Heiles et al. 1996). Heiles also claims that most shell structures occur beyond the solar circle. Here the star-formation rate is reduced compared with the inner disk, weakening the argument that young, massive stars provide the appropriate driving mechanism (although a lower stellar density in these regions might allow gas to escape more easily from the disk).

In contrast to the Milky Way, HI observations of the face-on spiral NGC 6946 (Kamphuis \& Sancisi 1993) have established a link between high-velocity atomic gas and OB associations in the disk. The material, travelling at $z$ velocities of up to $100 \mathrm{~km} \mathrm{~s}^{-1}$, occurs over holes in the HI disk where young stars have driven material upwards. The ejected mass in these "vertical" structures is somewhat higher than the values we record for individual chimneys in the R96 sample $\left(10^{7} M_{\odot}\right.$ cf. $10^{5}-10^{6} M_{\odot}$ in Table 4$)$. However, in total, the quantity of extraplanar material in question is remarkably similar in both cases $(\sim 2 \%$ of $\mathrm{HI}$ gas in NGC 6946 is believed to be displaced from the main disk).

Whilst detected at high latitudes, the Galactic cirrus is said to correlate well with local molecular gas clouds (Weiland et al. 1986). This material is typified by distances and $z$-heights of order $100 \mathrm{pc}$ (rather than kpc). The opacity associated with molecular cirrus clouds is $\sim 1$ in $\tau_{B}$ i.e. close to the optical depth derived for dust chimneys. For cirrus coupled to atomic gas clouds the opacity is much lower $\left(\tau_{B} \sim 0.1\right.$; Low et al. 1984). Likewise, measurements of interstellar reddening towards $\mathrm{A}$ and $\mathrm{F}$ stars in the solar neighborhood suggest only small amounts of high- $z$ dust at least locally (e.g. $A_{B}<0.1$ mag above the sun; Burstein \& Heiles 1982). In contrast, Davies et al. (1997) found that the 140 and $240 \mu \mathrm{m}$ emission detected by COBE indicated a much more vertically extended dust distribution. Indeed, they suggest a scaleheight of $500 \mathrm{pc}$ for diffuse Galactic dust i.e. twice the scale-height of the stellar disk and 4 times greater than the conventional extinction layer. The method employed by Davies et al. 
consisted of fitting COBE FIR maps, in a latitudelongitude reference frame, with a dust disk of exponential fall-off in radius and $z$-height. Given the limited perspective, however, it is possible that the global fit might have been easily biased by local cirrus features (the nearby North Polar Spur, for example, appear prominently in the COBE images).

In conclusion, there is less compelling evidence for high- $z$ dust in the Galaxy compared with spirals such as NGC 891. However, we recognize that our "in-plane perspective" of the Milky Way disk may either distort or impair our perception of extraplanar material. The existence of HI shells with scale-heights of $1 \mathrm{kpc}$ could indicate that dust chimneys, similar to those found in the R96 sample, are also present in the Galaxy.

\subsection{Mass loss and intergalactic enrichment}

The rate at which dust and heavy elements is expelled from the stellar disk may depend critically on the nature of the outflow mechanism. For example, if grains are driven primarily by gas pressure it may be appropriate to use the expansion velocity of the neutral gas out of the disk. The velocities recorded for Heiles' supershells are typically no more than $50 \mathrm{~km} \mathrm{~s}^{-1}$. The high velocity neutral gas detected in NGC 6946 is believed to be moving up $100 \mathrm{~km} \mathrm{~s}^{-1}$ away from the disk. Norman \& Ikeuchi (1989) describe a scenario whereby the concentration of Type II SNe in Galactic OB associations should, in principle generate the kind of superbubble structures observed by Heiles. The chimney structures thereby constitute the dense, fragmented walls of the bubble as the expanding cavity bursts and hot gas from the OB association escapes into the halo. The expected timescale for this outflow, which assumes that the dust and neutral gas are entrained at the working surface of the superbubble, is $\sim 10^{7} \mathrm{yr}$. This value is consistent with an expulsion velocity of $100 \mathrm{~km} \mathrm{~s}^{-1}$ as observed for the neutral gas in NGC 6946.

As mentioned previously, there exists the strong possibility that extraplanar dust is propelled preferentially by radiation pressure rather than gas convective flow. Ferrara (1998) has carried out a number of simulations in which grains above a typical OB association form chimney-like structures around a cavity swept clean of dust by intense radiation pressure. The journey time is once again a few $\times 10^{7}$ yr with grains attaining upward velocities of $\sim 100 \mathrm{~km} \mathrm{~s}^{-1}$. In addition to gravitational and radiative forces, the Ferrara calculations attempt to take account of viscous and coulomb drag on the grains, the latter requiring some knowledge of the grain charge. In a similar vein, Davies et al. (1998) estimated the radiation pressure on various sizes of grains due to the general interstellar radiation field from the disk. Their model incorporated dark matter into the gravitational field and accounted for the fact that the grains themselves limit the distance over which photons can travel before being absorbed (self-shielding effect). The expulsion timescale, in this case, was $\sim 10^{8}$ yr for classical $0.1 \mu \mathrm{m}$ grains with a terminal velocity, $\sim 10 \mathrm{~km} \mathrm{~s}^{-1}$, controlled by the ambient gas density in the disk. The authors neglected the concentrated input of copious radiation from OB associations as well as the significant influence of magnetic fields on the grain movement.

The notion that radiation pressure might be responsible for the appearance of dust chimneys also raises some difficult issues. The absorption properties of extraplanar dust filaments are consistent with an optical depth of around unity along their shorter axis. Thus the middle of such structures might be significantly shielded from optical radiation propogating upwards from the disk. If the dust filament is, indeed, self-shielded in this sense, it is difficult to see how such structures can be pushed outwards via radiation pressure. Furthermore, the vertical dust features appear remarkable well-contained, in comparison with the DIG for example. Are such clearly-demarcated structures likely to arise naturally if grains are propelled by radiation pressure rather than gas pressure from the disk? A strong possibility is that dust grains are constrained to move along magnetic field lines due to their electric charge. Upwelling, ionized gas from star-forming regions in the disk appears capable of producing buckles in the toroidal magnetic field. This generates, over local regions of the disk $(100 \mathrm{pc} \times 100 \mathrm{pc})$, a magnetic field orientated perpendicular to the disk. Such a phenomenon may prevent grains from dispersing during their upward passage, facilitating, in effect, the formation of vertical dust lanes (see Sofue et al. 1994 for a discussion).

In our estimate of dust seepage from the stellar disk we use an upward maximum velocity of $100 \mathrm{~km} \mathrm{~s}^{-1}$. This is in keeping with velocities observed for HI supershells in the Milky Way and NGC 6946 and, at the same time, remains consistent with the radiation pressure simulations carried out by Ferrara. The typical escape velocity from a large spiral disk is a few $\times 100 \mathrm{~km} \mathrm{~s}^{-1}$ (Phillips 1993), somewhat greater than our estimated upward expansion. It seems unlikely then that dust can breach the halo into the intergalactic medium (IGM), unless it is caused to accelerate at greater $z$-height. An increase in acceleration does not seem plausible, however, because the respective forces due to gravity and radiation pressure are both governed by the same $1 / r^{2}$ form. Thus, the balance of these two forces on the ascending grain is more or less fixed, for all $z$-heights, by the mass-to-light ratio of the disk (in fact, as the grain rises, it is the radiation pressure that diminishes preferentially due to increasing obscuration of disk starlight). Our unsharp-mask images of the R 96 sample, do not show any evidence for dust travelling to more than $2 \mathrm{kpc}$ above the midplane. As already pointed out, however, techniques which employ extinction to locate extraplanar grains are rather insensitive to tenuous dust clouds 
situated so far above the stars that they are unable to produce well-defined absorption features. Some of the chimney features in our data are suggestive of a reconnection with the main absorption layer. This conceivably has more to do with the grains being tied to magnetic field lines rather than a genuine "fountain" effect. As already mentioned, dust features may well follow "loops" and "buckles" in the main toroidal field allowing them to stretch upwards and then reconnect elsewhere with the disk.

Boyle et al. (1988) estimate that the IGM of the nearby Virgo Cluster systematically reddens background galaxies and QSOs by the equivalent of $\simeq 0.2$ in $A_{B}$. This attenuating material presumably originates from the cluster members. Integrating over the surface area of Virgo (4.4 $10^{6} \mathrm{kpc}^{2}$ ), the total dust mass residing outside the cluster members is expected to be large $\left(\sim 10^{11} M_{\odot}\right)$. A straightforward calculation shows that the dust chimneys within quiescent disks are unlikely to constitute such prodigious sources of dust (even if ejected grains acquire the appropriate escape velocity). We assume that half the 180 spirals in the Virgo Cluster possess vertical dust structures and that these "ducts" allow $1 \%$ of the disk's grain mass to enter the IGM over the course of $10^{7} \mathrm{yr}$. These assumptions are based on the inferences of outflow rate reported above and the amount of extraplanar dust detected in our R 96 sample. Under such circumstances, it would require $10^{11} \mathrm{yr}$ for outflows from conventional, quiescent disks to account for the dust accumulated in the Virgo IGM - a period well in excess of the cluster lifetime. Far more likely origins for intergalactic dust are "superwinds", as certain cluster members undergo intense periods of starburst activity, or, indeed, tidal interactions between neighboring galaxies (Wiebe et al. 1999; Alton et al. 1999a; Yun et al. 1993). Doyon \& Joseph (1989) also claim that ram-stripping may be an important dust removal mechanism amongst Virgo spirals. Here, the velocity of the cluster members with respect to the IGM is itself sufficient to strip loosely-bound gas and dust from the disk environment.

A corollary to the above calculation is that if chimneys deplete the ISM at a rate of $1 \%$ every $10^{7} \mathrm{yr}$, then the total grain content of quiescent disks $\left(510^{7} M_{\odot}\right)$ must be replenished on timescales of $10^{9}$ years. In fact, the current, best estimate of the rate at which stardust is added to the ISM is $\sim 0.04 M_{\odot} \mathrm{yr}^{-1}$ (Whittet 1992). This indeed points to a replenishment timescale of $10^{9}$ yr suggesting that dust chimneys could reasonably form part of the natural cycling and processing of interstellar material.

Although the chimneys we have been describing may not contribute significantly to the enrichment of the IGM with dust and metals, they are likely to play an important role in the circulation of heavy elements and gas around the disk. Any material that falls back to the disk will serve to homogenize the chemical make-up of the ISM allowing star-formation to proceed with greater efficiency than can be envisaged in a system with zero gas flow. Indeed, if models of chemical evolution are to successfully account for the efficiency of star formation in galactic disks a means of mixing the ISM must be supplied (Edmunds 1996; Edmunds \& Phillipps 1997). Finally, as far as the distribution of dust in spirals is concerned, there is certainly increasing observational evidence that grains are more widely distributed than the stars that are expected to produce them. On the basis of systematic reddening of background galaxies, Zaritsky (1994) claims that galactic dust extends out to a radius of $60 \mathrm{kpc}$ along the major axis of nearby spirals! Nelson et al. (1998) infer a more modest, but nonetheless very large, dust radial extent for nearby galaxies mapped in the $100 \mu \mathrm{m}$ IRAS Sky Survey $(20-30 \mathrm{kpc})$. Likewise, ISOPHOT $200 \mu \mathrm{m}$ images of cold dust $(15-20 \mathrm{~K})$ are indicative of dust scale-lengths $70 \%$ larger than that of the stellar disk (Alton et al. 1998c). For grains to occur at larger galactocentric distances than the "edge" of the stellar disk, either star-formation was more radial extensive in the past or galactic dust has been significantly displaced subsequent to its formation.

\section{Summary}

We use $B$-band CCD images to search for the presence of extraplanar dust or dust chimneys in a sample of 10 edgeon, spiral galaxies which we had previously observed in the $\mathrm{H} \alpha$ emission line (Rand 1996). The existence of such features in nearby NGC 891 has been known for some time. However, their overall occurence in quiescent (nonstarburst) disks, as well as the manner in which they are formed, has only come under investigation very recently. We quantify the amount of extraplanar dust by identifying (with the aid of unsharp-masking) extinction features which cannot be accounted for by the standard absorption dust lane. This can only be done reliably for 5 of the most edge-on galaxies in our sample $\left(i>87^{\circ}\right)$ and we use a scattering +absorption radiation-transfer model (Xilouris et al. 1999) to separate "vertical" extinction features from the conventional dust disk in these cases. We investigate the possibility that dust is expelled from stellar disks by localized star-formation by comparing the $B$-band unsharpmask frames with our $\mathrm{H} \alpha$ images. Our main conclusions from this study are as follows:

1. Of the 5 galaxies deemed sufficiently edge-on to warrant a search for extraplanar dust, 3 show strong evidence for dust chimneys and isolated high- $z$ extinction clouds. The linear features extend up to $2 \mathrm{kpc}$ above the midplane with some simply terminating at high latitudes and others reconnecting with the disk. In general, the extraplanar features are poorly resolved in our images with the consequence that the opacities we derive $\left(\tau_{B} \simeq 0.15\right)$ are too low by a factor of 5 or so. The masses we recover for the individual dust structures are expected to be correct and are 
of order $10^{5} M_{\odot}$ (assuming a solar-type gas-to-dust ratio). With this technique, the total amount of dust we find outside the standard dust lane is $\sim 1 \%$;

2. If we combine our results with a very recent similar study by Howk \& Savage (1999), we believe that 5 out of the 10 disks, which are sufficiently inclined to allow us to probe the extraplanar layer $(i>87)$, possess high-lattitude dust. We concur with Howk \& Savage that the "chaotic" dust lane in NGC 891 cannot be considered anomalous and vertical chimneys probably occur in half of all quiescent spiral galaxies;

3. Our use of optical extinction to identify extraplanar dust is heavily biassed towards dust clouds on the nearside of the disk and relies on sufficient background light from the bulge to make attenuation conspicuous. Consequently, we explore the transparent submillimeter thermal emission recently detected from cold dust in NGC 891. Profiles in z-height, at 450 and $850 \mu \mathrm{m}$, constrain the quantity of extraplanar dust to $<5 \%$ of the total galactic dust mass;

4. We can establish no detailed spatial correlation between dust chimneys and either $\mathrm{H} \alpha$ emission emanating from the disk (HII regions) or the near-halo environment (Diffuse Ionized Gas). This is something of a surprise since far-infrared bright galaxies (such as $\mathrm{M}$ 82) are known to entrain optically-thick dust at the working surface of the starburst outflow. The Diffuse Ionized Gas in quiescent galaxies is believed to arise from $\mathrm{O}$-stars in HII regions keeping high-latitude $(z=1-4 \mathrm{kpc})$ gas in a state of ionization. Indeed, for our edge-on sample, brightest Diffuse Ionized Gas emission occurs above areas of the disk experiencing relatively high rates of recent star formation. There is also some possibility that we find dust chimneys occurring more frequently in galaxies with higher overall star-forming activity. The fact that recent starformation and extraplanar dust are not coupled locally (but might correlate globally) may suggest a difference in timescale between the two phenomena. Chimneys probably take $\sim 10^{7}$ yr to form, regardless of whether they are driven out by radiation of gas pressure. On the other hand, $\mathrm{H} \alpha$ emission traces O-stars and these massive stars are known to possess lifetimes of $\sim 10^{6}$ yr;

5. We examine $\mathrm{H} \alpha$ and optical images of the nearby edgeon disk galaxy NGC 55. Its proximity $(D=1.6 \mathrm{Mpc})$ offers vastly superior resolution over galaxies comprising our main sample. The most active star-forming region near the centre of NGC 55 appears to have prised dust clouds away from the midplane. However, more generally, we find no obvious correlation between local disturbances in the dust lane and increased $\mathrm{H} \alpha$ emission;

6. We investigate the possibility that the Milky Way might contain extraplanar dust. It is conceivable that the supershell structures described by Heiles may re- late to dust chimneys. However, there is little evidence for high- $z$ dust from observations of local cirrus and reddening measured towards local low mass stars;

7. Dust chimneys are unlikely to be significant contributors to intergalactic enrichment. Dust and metals detected in clusters such as Virgo and Coma can probably be attributed to phenomena such as starburst superwinds, tidal interactions and ram-stripping. Despite this, dust chimneys must play an important role in the circulation of gas, grains and heavy elements around the interstellar medium. Chemical mixing of this kind allows star-formation to proceed efficiently within spiral disks.

Acknowledgements. We thank the archival services of the ING and AAO telescopes for making CCD data available to us so quickly. We are also grateful to the IRAS archivists at IPAC, Caltech. PBA acknowledges his continued financial support from PPARC.

\section{References}

Allen R., Baldwin J., Sancisi R., 1978, A\&A 62, 397

Alton P.B., Bianchi S., Rand R., et al., 1998a, ApJL 507, L125

Alton P.B., Davies J., Trewhella M., 1998b, MNRAS 296, 773

Alton P.B., Trewhella M., Davies J., et al., 1998c, A\&A 335, 807

Alton P.B., Davies J., Bianchi S., 1999a, A\&A 343, 51

Alton P.B., Xilouris E., Bianchi S., Davies J., Kylafis N., 1999b, A\&A (submitted)

Bahcall J., 1983, ApJ 267, 52

Bianchi S., Ferrara A., Giovanardi C., 1996, ApJ 465, 127

Bohlin R., Savage B., Drake J., 1978, ApJ 224, 132

Bohren C., Huffman D., 1983, in Absorption \& Scattering of light by Small Particles. John Wiley \& Sons, New York

Bomans D., Grebel E., 1993, in Violent Star Formation, Tenorio-Tagle G. (ed.). Cambridge University Press, Cambridge, p. 156

Boyle B., Fong R., Shanks T., 1988, MNRAS 231, 77

Bregman J., Pildis R., 1994, ApJ 420, 570

Burstein D., Heiles C., 1982, AJ 87, 1165

Chevalier T., Clegg A., 1985, Nat 317, 44

Crutcher R., 1982, ApJ 254, 82

Davies J., Trewhella M., Jones H., et al., MNRAS 1997, 288, 679

Davies J., Alton P., Bianchi S., Trewhella M., 1998, MNRAS 300, 1006

de Vaucouleurs G., de Vaucouleurs A., Corwin H., et al., 1991, Third Reference Catalogue of Bright Galaxies (RC3)

Dettmar R.-J., 1990, A\&A 232, L15

Devereux N., Young J., 1990, ApJ 359, 42

Devereux N., Young J., 1993, AJ 106, 948

Doyon R., Joseph R., 1989, MNRAS 239, 347

Dressler A., 1980, ApJ 236, 351

Edmunds M., 1996, in Burkert A., Hartmann D., Majewski S. (eds.) Galactic Chemodynamics: The History of the Milky Way and its Satellite Systems. Astronomical Society of the Pacific

Edmunds M., Phillipps S., 1997, MNRAS 292, 733

Fall S., Pei Y., 1993, ApJ 402, 479 
Ferrara A., 1998, IAU Symp. 166, 371

Ferguson A., Wyse R., Gallagher J., AJ 112, 2567

Garcia-Burillo S., Guelin M., Cernicharo J., Dahlem M., 1992, A\&A 266, 21

Gerola A., Seiden P., Schulman L., 1980, ApJ 242, 517

Guelin M., Zylka R., Mezger P., et al., 1993, A\&A 270, L37

Heckman T., Armus L., Miley G., 1990, ApJS 74, 833

Heiles C., Chu Y.-H., Reynolds R., Yegingil I., Troland T., 1980, ApJ 242, 533

Heiles C., 1984, ApJS 55, 585

Heiles C., Reach W., Koo B., 1996, ApJ 466, 191

Heisler J., Ostriker J., 1988, ApJ 332, 453

Henyey L., Greenstein J., 1941, ApJ 93, 70

Howk J., Savage B., 1997, AJ 114, 2463 (HS97)

Howk J., Savage B., 1999, AJ 117, 2077 (HS99)

Hu E., 1981, ApJ 248, 119

Hughes D., Gear W., Robson E., 1994, MNRAS 270, 641

Hunter D., Hawley W., Gallagher J., 1993, AJ 106, 1797

Ichikawa T., van Driel W., Aoki T., et al., 1994, ApJ 433, 645

Israel F., van der Werf P., Tilanus R., 1999, A\&A 344, L831

Kamphuis J., Sancisi R., 1993, A\&A 273, L31

Keppel J., Dettmar R.-J., Gallagher J., 1991, ApJ 374, 507

Kulkarni S., Heiles C., 1988, in Galactic and Extragalactic Radio Astronomy, Verschuur G. \& Kellermann K. (eds.). New York, Springer, p. 95

Kunth D., Maurogordato S., Vigroux L., 1988, A\&A 204, 10

Lanzetta K., Trunshek D., Wolfe A., 1987, ApJ 322, 739

Leitherer C., Robert C., Drissen L., 1992, ApJ 401, 596

Leitherer C., Heckman T., 1995, ApJS 96, 9

Malhotra S., 1997, ApJ 488, L101

Marlowe A., Heckman T., Wyse R., Schommer R., 1995, ApJ 438,563

Masci F., Webster R., 1999, MNRAS 305, 937

Mathis J., 1990, ARA\&A 28, 37
McCarthy P., Heckman T., van Breugel W., 1987, AJ 93, 264

Meaburn J., 1980, MNRAS 192, 365

Nelson A., Zaritsky D., Cutri R., 1998, AJ 115, 2273

Norman C, Ikeuchi S., 1989, ApJ 345, 372

Phillips A., 1993, AJ 105, 486

Puche D., Cariganan C., Wainscoat R., 1991, AJ 101, 447

Rand R., Kulkarni S., Hester J., 1990, ApJ 352, L1

Rand R., 1996, ApJ 462, 712 (R96)

Rand R., 1998, ApJ 501, 137

Reynolds R., 1992, ApJ 392, L35

Sancisi R., Allen R., 1979, A\&A 74, 73

Scoville N., Thakkar D., Carlstrom J., Sargent A., 1993, ApJ 404, 53

Sofue Y., Wakamatsu K., Malin D., 1994, AJ 108, 2102

Soifer B., Sanders D., Madore B., et al., 1987, ApJ 320, 238

Swaters R., Sancisi R., van der Hulst J., 1997, ApJ 491, 140

Vacca W., Conti P., 1992, ApJ 401, 543

van Albada T., Bahcall J., Begeman K., Sanscisi R., 1985, ApJ 295,305

Walterbos R., Greenawalt B., 1996, ApJ 460, 696

Webster R., Francis P., Peterson B., Drinkwater M., Masci F., 1995, Nat 375, 469

Weiland J., Blitz L., Dwek E., et al., 1986, ApJ 306, L101

Whittet D., 1992, Dust in the Galactic Environment. IOP Publishing

Wiebe D., Shustov B., Tutukov A., A\&A 345, 93

Witt A., Gordon K., Madsen G., 1999, ApJ (in press)

Xilouris E., Kylafis N., Papamastorakis J., Paleologou E., Haerendel G., 1997, A\&A 325, 135

Xilouris E., Alton P., Davies J., et al., 1998, A\&A 331, 894

Xilouris E., Byun Y., Kylafis N., Paleologou E., Papamastorakis J., 1999, A\&A 344, 868

Yun M., Ho P., Lo K., 1993, ApJ 411, L17

Zaritsky D., 1994, AJ 108, 1619 